\title{
Swi/Snf dynamics on stress-responsive genes is governed by competitive bromodomain interactions
}

\author{
Arnob Dutta, ${ }^{1}$ Madelaine Gogol, ${ }^{1}$ Jeong-Hoon Kim, ${ }^{2}$ Michaela Smolle, ${ }^{1}$ Swaminathan Venkatesh, ${ }^{1}$ \\ Joshua Gilmore, ${ }^{1}$ Laurence Florens, ${ }^{1}$ Michael P. Washburn, ${ }^{1,3}$ and Jerry L. Workman ${ }^{1}$ \\ ${ }^{1}$ Stowers Institute for Medical Research, Kansas City, Missouri 64110, USA; ${ }^{2}$ Medical Proteomics Research Center, Korea \\ Research Institute of Bioscience and Biotechnology, Yuseong-gu, Daejeon 305-806, Korea; ${ }^{3}$ Department of Pathology and \\ Laboratory Medicine, University of Kansas Medical Center, Kansas City, Kansas 66160, USA
}

\begin{abstract}
The Swi/Snf chromatin remodeling complex functions to alter nucleosome positions by either sliding nucleosomes on DNA or the eviction of histones. The presence of histone acetylation and activator-dependent recruitment and retention of Swi/Snf is important for its efficient function. It is not understood, however, why such mechanisms are required to enhance Swi/Snf activity on nucleosomes. Snf2, the catalytic subunit of the Swi/Snf remodeling complex, has been shown to be a target of the Gen5 acetyltransferase. Our study found that acetylation of Snf2 regulates both recruitment and release of Swi/Snf from stress-responsive genes. Also, the intramolecular interaction of the Snf2 bromodomain with the acetylated lysine residues on Snf2 negatively regulates binding and remodeling of acetylated nucleosomes by Swi/Snf. Interestingly, the presence of transcription activators mitigates the effects of the reduced affinity of acetylated Snf2 for acetylated nucleosomes. Supporting our in vitro results, we found that activator-bound genes regulating metabolic processes showed greater retention of the Swi/Snf complex even when Snf2 was acetylated. Our studies demonstrate that competing effects of (1) Swi/Snf retention by activators or high levels of histone acetylation and (2) Snf2 acetylation-mediated release regulate dynamics of Swi/Snf occupancy at target genes.
\end{abstract}

[Keywords: Swi/Snf; activator; histone acetylation; stress]

Supplemental material is available for this article.

Received April 14, 2014; revised version accepted September 5, 2014.

Packaging of DNA into higher-order chromatin acts as a barrier to transcription. Accessibility to the underlying DNA sequences can be facilitated by the coordinated action of chromatin-modifying enzymes and ATP-dependent chromatin remodelers, which post-translationally modify histones or use ATP hydrolysis to slide or evict nucleosomes, respectively. The action of these coactivators allows temporal and spatial regulation of transcription. The targeting and activity of chromatin remodeling enzymes need to be tightly regulated. The multisubunit Swi/Snf complex functions in the regulation of stress response genes as well as activation of gene expression during normal growth conditions (Sudarsanam and Winston 2000; Sudarsanam et al. 2000; Shivaswamy and Iyer 2008). Swi/Snf occupancy at the promoters of genes has been shown to be regulated by activator-mediated targeting and retention along with SAGA-dependent acetylation of nucleosomes (Hassan et al. 2002, 2006; Neely et al. 2002; Prochasson et al. 2003; Chandy et al. 2006; Gutierrez et al. 2007). The presence of transcription activators also

Corresponding author: jlw@stowers.org

Article is online at http://www.genesdev.org/cgi/doi/10.1101/gad.243584.114. significantly increases the sliding of nucleosomes and eviction of histones by the Swi/Snf complex.

In recent years, studies have highlighted the role of posttranslational modifications of remodeling complexes in regulating their function. Interestingly, acetyltransferase complexes that were initially identified as modifiers of histones have also been shown to modify nonhistone proteins. Gcn5-the acetyltransferase in SAGA, SLIK, and ADA complexes (Lee and Workman 2007)—regulates activity of the RSC complex during replication stress by acetylation of the Rsc4 subunit (VanDemark et al. 2007; Charles et al. 2011). Also, Gcn5 acetylates ISWI in Drosophila (Ferreira et al. 2007) and proliferator $\gamma$ coactivator 1 (PGC-1) in mammals (Lerin et al. 2006). More recently, we showed that Snf2, the catalytic subunit of Swi/Snf, is acetylated by Gon5 (Kim et al. 2010). Interestingly, the Snf2 bromodomain, which is known to contact

(C) 2014 Dutta et al. This article is distributed exclusively by Cold Spring Harbor Laboratory Press for the first six months after the fullissue publication date (see http://genesdev.cshlp.org/site/misc/terms. xhtml). After six months, it is available under a Creative Commons License (Attribution-NonCommercial 4.0 International), as described at http://creativecommons.org/licenses/by-nc/4.0/. 
acetylated residues on histone tails (Hassan et al. 2002, 2006), binds intramolecularly to the Snf2 AT-hook region upon its acetylation. Such an interaction would suggest competition between acetylated histone tails and acetylated residues in Snf2 for binding the bromodomain, which might necessitate the presence of accessory factors for the recruitment and functioning of the Swi/Snf complex. However, what role acetylation of Snf2 plays in regulating biochemical activities of the Swi/Snf complex on nucleosomal templates in vitro and its effects on Swi/Snf occupancy at a genome-wide scale have not been addressed. Also, it remains to be seen how activator-dependent regulation of Swi/Snf activities and genome-wide recruitment are affected by acetylation of Snf2.

Here, we characterized the effects of Snf2 acetylation on biochemical activities of the Swi/Snf complex and performed genome-wide analyses of Swi/Snf occupancy. We found that acetylation of Snf2 reduces interaction of Swi/Snf with acetylated nucleosomes, resulting in reduced remodeling of acetylated nucleosome substrates with no effects on its ATPase activity. Interestingly, the addition of activator Gal4-VP16 stimulates binding and remodeling of Swi/Snf with acetylated Snf2 to levels similar to that when Snf2 is not acetylated. Indeed, genome-wide analysis of Swi/Snf occupancy supports our in vitro results in which we found that Swi/Snf-regulated genes controlling key cellular and metabolic processes have moderate levels of histone H3K9Ac but are activator-bound, which allows greater retention of the Swi/Snf complex despite acetylation of Snf2. On the other hand, acetylation of Snf2 regulates both recruitment and release of Swi/Snf from the promoters of stress-responsive genes regulated by nutrient deprivation, many of which also show high levels of histone acetylation. Snf2 acetylation-mediated release of Swi/Snf is also important to alter nucleosome positioning, especially at genes that are down-regulated during nutrient deprivation-induced stress. We further found that loss of the Snf2 bromodomain reduces Snf2 occupancy at stress-regulated genes. This is in agreement with observations that acetylation of Snf2 can modulate interactions of the Snf2 bromodomain with acetylated nucleosomes. Together, these modes of regulation act independently or in concert to target Swi/Snf at promoters such that activators or increased levels of histone acetylation are needed to mitigate the effects of acetylation of Snf2. Our studies demonstrate that acetylated Snf2 plays a role in dynamic regulation of gene functions by modulating distribution of the Swi/Snf complex to cope with changes during stress conditions.

\section{Results}

Snf2 acetylation regulates occupancy of Swi/Snf during stress

$\mathrm{Swi} / \mathrm{Snf}$ is recruited to $\sim 5 \%$ of all yeast genes under normal growth conditions (Yen et al. 2012) but, more importantly, regulates transcription of stress response genes (Peterson and Workman 2000; Sudarsanam and
Winston 2000; Geng and Laurent 2004; Shivaswamy and Iyer 2008). To study how Snf2 acetylation affected Swi/Snf occupancy genome-wide, the wild type and the acetylation site mutant Snf2 K1493R K1497R (2R) were grown in rich medium with $2 \%$ dextrose, which served as an unstressed condition in our experiments. To induce stress, cells grown in rich medium were transferred to minimal medium without amino acids and $0.05 \%$ dextrose for $10 \mathrm{~min}$. Here we refer to this specific conditionacute deletion of glucose and amino acids-as the stress condition. Chromatin immunoprecipitation (ChIP) was carried out using Snf2 antibody, followed by highthroughput sequencing (ChIP-seq). Genome average plots under both growth conditions showed greater occupancy around the promoter compared with the body of genes (Fig. 1A,B). During unstressed conditions, no significant change in occupancy was observed between the wild type and $2 \mathrm{R}$ upstream of the transcription start site (TSS), but increased occupancy was observed over the ORF for the 2R mutant (pval $<0.005$ over the ORF) (Fig. 1A). Interestingly, upon induction of stress, Swi/Snf occupancy was significantly increased in the $2 \mathrm{R}$ mutant compared with wild type at both the promoter and the ORF $($ pval $<0.001$ over both the ORF and 500 base pairs [bp] upstream of the TSS), suggesting that Snf2 acetylation may be involved in regulating levels of Swi/Snf occupancy on a global scale (Fig. 1B).

As a response to environmental stress, different subsets of genes are turned on or shut off (Gasch et al. 2000; Causton et al. 2001; Gasch and Werner-Washburne 2002). To address how acetylation affected dynamics of Swi/Snf occupancy, Snf2-bound genes were characterized into three groups based on changes in Snf2 occupancy during normal or stress growth conditions (Fig. 1C). Group 1 genes showed a reduction of Swi/Snf occupancy upon stress. Group 1 genes, which were enriched for translation and ribosome synthesis genes (Supplemental Fig. 1a), showed similar levels of Swi/Snf occupancy in the wild type and 2R under normal growth conditions (Figs. 1C, 2A). During stress, Swi/Snf was depleted from both promoters and gene bodies in the case of wild type (Figs. 1C, 2B,C), but the 2R mutant showed greater retention of Swi/Snf at the promoters, although levels at the ORF were similar to the wild type. Conversely, group 2 genes showed an increase of Swi/Snf occupancy upon stress. Group 2 genes-with gene ontology (GO) term enrichment for genes involved in carbohydrate metabolism, stress response, and amino acid synthesis (Supplemental Fig. 1a)-had low occupancy of Swi/Snf under unstressed conditions (Figs. 1C, 2D). Upon stress, Swi/Snf occupancy increased at these genes in both cases; however, $2 \mathrm{R}$ showed greater occupancy at both the promoter and the ORF (Figs. 1C, 2E,F). Group 3 genes were occupied by Swi/Snf under both conditions. Group 3 genes, with GO term enrichment for genes involved in metabolic processes (Supplemental Fig. 1a), showed no significant differences in levels of Swi/Snf occupancy between the wild type and mutant under unstressed conditions but increased occupancy in 2R during stress (Figs. 1C, 2G-I). This most likely 
Dutta et al.
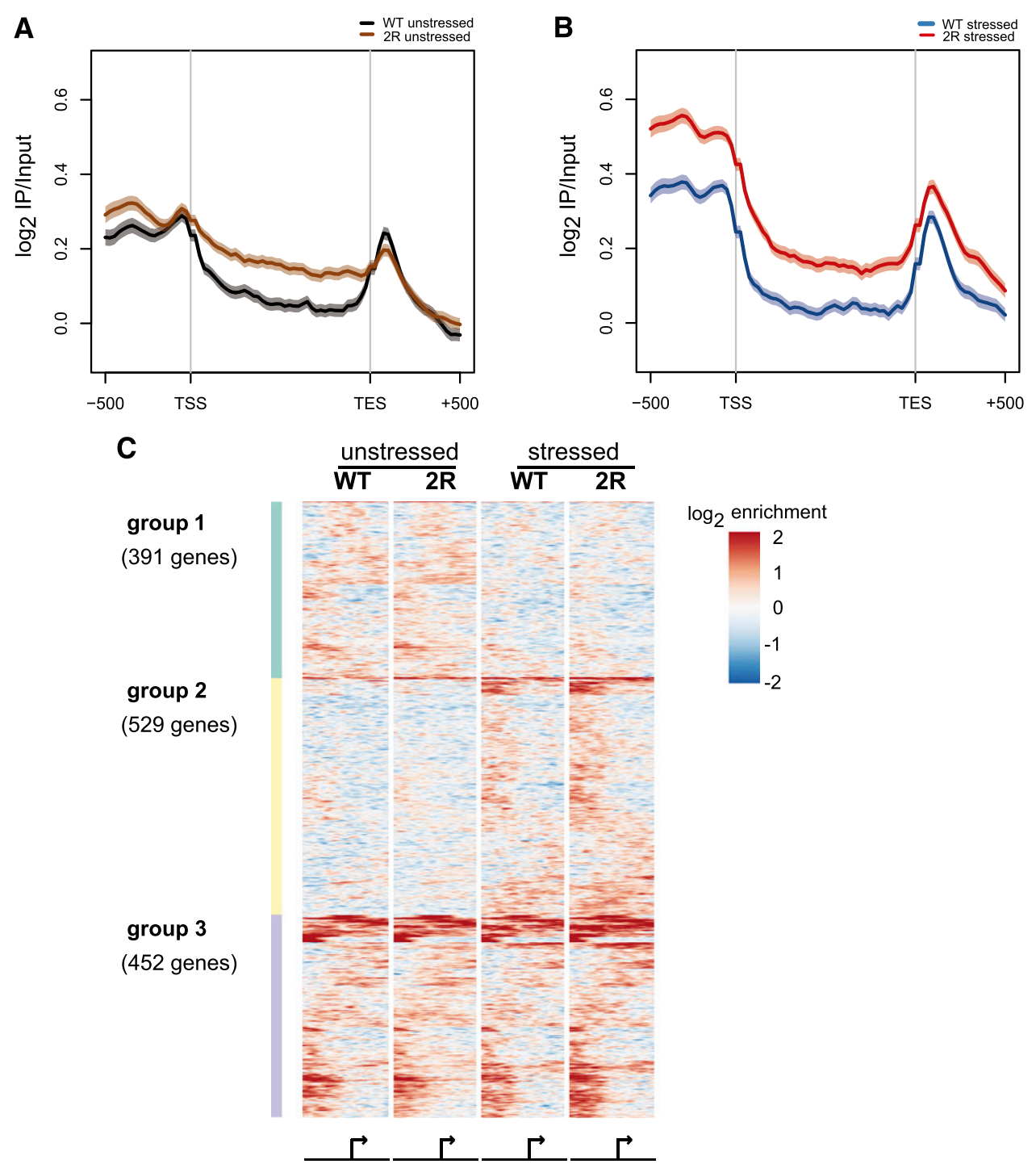

Figure 1. Swi/Snf occupancy at genes under stressed and unstressed conditions. (A) Occupancy of Snf2 under unstressed conditions. (B) Occupancy of Snf2 under stressed conditions. ChIP experiments were performed using Snf2 antibody in wild-type (WT) and Snf2 K1493R K1497R (2R) strains. $\log _{2}$ ratios of normalized enrichment (immunoprecipitation [IP]/input) for each gene region, including $500 \mathrm{bp}$ upstream of and downstream from the gene, were used for average gene analysis. Genome average data of genes that showed enrichment of at least 1.7-fold of immunoprecipitation/input in both replicates were calculated and plotted. The TSS and termination site (TES) are indicated. Transparent shading around the line indicates the standard error of the mean. (C) Genes were grouped based on changes in Snf2 occupancy during stress. The heat map of Snf2 occupancy for wild type and Snf2 K1493R K1497R (2R) in each group under stressed and unstressed conditions was generated by hierarchically row-clustering normalized $\log _{2}$ (immunoprecipitation/input) values for regions $-700 /+500$ around the TSS of genes.

represents an increase in expression of a subset of these genes during stress. These results point to the fact that loss of Snf2 acetylation slows down release of Swi/Snf from genes repressed by stress while increasing the levels of Swi/Snf recruitment at genes up-regulated by stress.

\section{Acetylation of Snf2 modulates Swi/Snf binding and remodeling of acetylated nucleosomes}

Our genome-wide analysis suggested that Snf2 acetylation plays a role in recruitment and retention of Swi/Snf at target genes. To test the biochemical basis for differences in Swi/Snf occupancy, we tested binding of Swi/Snf to DNA and nucleosomes. The analysis of purified Swi/Snf complexes-wild type, 2R, and from $\Delta g c n 5$ strains (Snf2 would be unacetylated in a $\Delta g c n 5$ mutant) (Kim et al. 2010)-showed no differences in complex composition by silver staining (Supplemental Fig. 2b) or mass spectrometry (data not shown). Western blot analysis confirmed that Snf2 was acetylated only in the wild type (Supplemental Fig. 2b). Furthermore, we carried out quantitative mass spectrometric analysis of the Swi/Snf complex purified from the wild type that was grown in YPD in order to determine what fraction of Snf2 was acetylated at residues K1493 and 
group 1

A
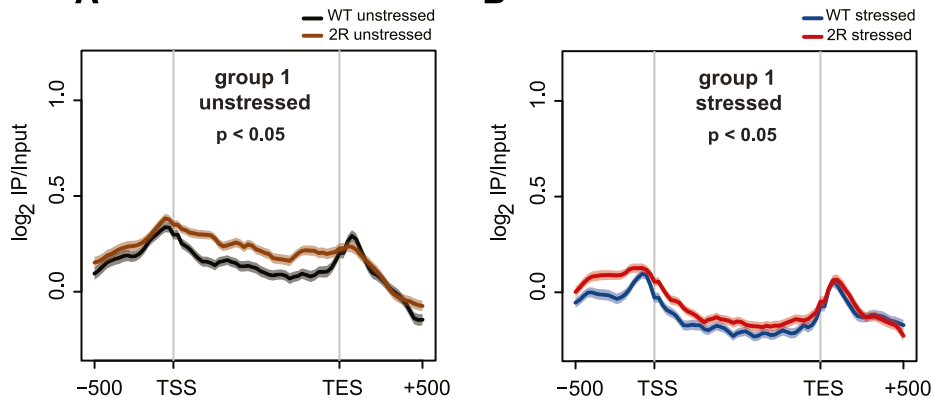

C

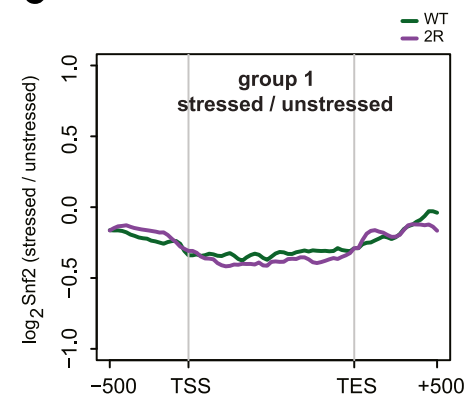

group 2

D

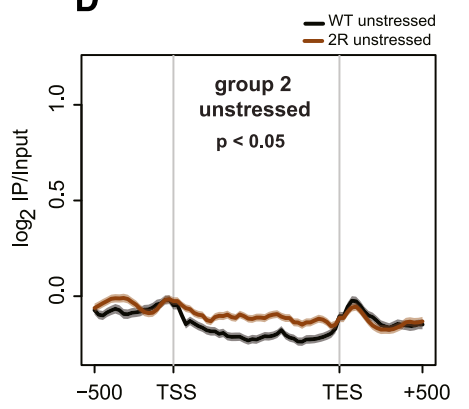

$\mathbf{E}$

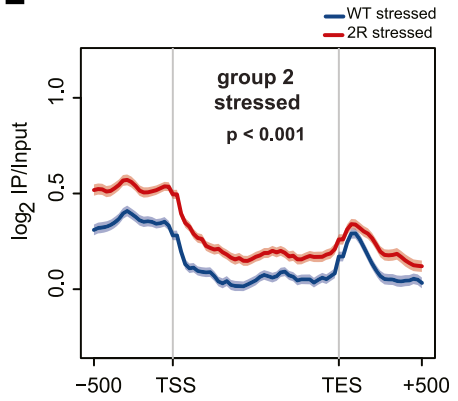

$\mathbf{F}$

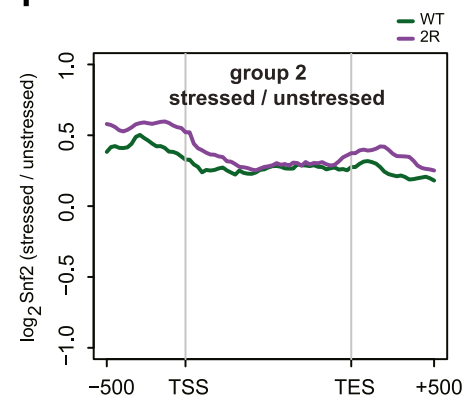

group 3
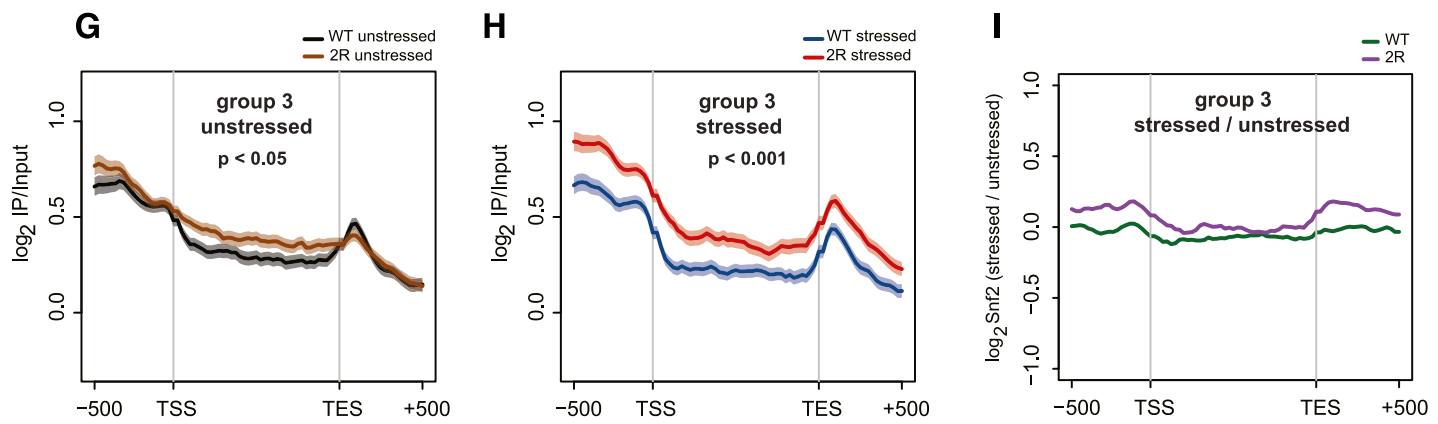

Figure 2. Acetylation of Snf2 regulates recruitment and release of Swi/Snf from genes during stress. $(A, B)$ Group 1: Genes $(391)$ at which Swi/Snf is occupied under unstressed conditions but reduced during stress. $(D, E)$ Group 2: Genes (529) that are not occupied by Swi/Snf during unstressed conditions, but their occupancy increases during stress. $(G, H)$ Group 3: Genes (452) that are occupied by Swi/ Snf under both unstressed and stress conditions. Average $\log _{2}$ ratios of normalized enrichment (immunoprecipitation [IP]/input) for each gene region, including $500 \mathrm{bp}$ upstream of and downstream from the gene, were calculated and plotted for both the wild type (WT) and Snf2 K1493R K1497R (2R) under unstressed and stressed conditions. The TSS and TES are indicated. Transparent shading around the line indicates the standard error of the mean. $P$-values are based on mean values per gene in the TSS region $(-700 /+500)$ and are from a two-sided Student's $t$-test between samples. $(C, F, I)$ Average $\log _{2}$ ratios of changes in Snf2 occupancy during stressed conditions for each gene region, including $500 \mathrm{bp}$ upstream of and downstream from the gene, were calculated and plotted for both the wild type and Snf2 K1493R K1497R (2R) for each group.

K1497. Approximately $70 \%$ of all peptides containing residues K1493 and K1497 were found to be acetylated (Supplemental Fig. 2c). Control purifications from a $\Delta g \mathrm{cn} 5$ strain showed that only $\sim 10 \%$ of both lysine residues were acetylated.

The sites of Snf2 acetylation lie between the two AThook domains (Supplemental Fig. 2a; Kim et al. 2010). Since AT hooks can interact with DNA (Singh et al.
2006), loss of positive charges due to acetylation of lysine residues could affect binding of Swi/Snf to DNA. Testing for DNA binding as a function of complex concentration showed no differences in binding between wild type and mutants (Supplemental Fig. 3a).

Since Swi/Snf functions by binding and remodeling nucleosomes (Gutierrez et al. 2007), we tested binding to mononucleosomes reconstituted on 601 positioning se- 
quence-containing DNA with linkers (25N47). Using Xenopus laevis histones purified from Escherichia coli ensured the absence of post-translational modifications. Wild-type and mutant complexes bound these unmodified nucleosomes with equal affinity (Fig. 3A; Supplemental Fig. $3 \mathrm{~b})$. Since Swi/Snf binding to these nucleosomes could be due to recruitment through linker DNA, we tested binding to $8 \mathrm{~N} 10$ nucleosomes with shorter linkers (Supplemental Fig. 3c) and to nucleosomes reconstituted on minimal 601 positioning sequence without linker DNA (data not shown). We did not observe any differences in binding between wild-type and mutant Swi/ Snf. Thus, acetylation of Snf2 does not affect binding of Swi/Snf to nucleosomes without post-translational modifications.

SAGA-mediated acetylation of nucleosomes increases recruitment and remodeling by Swi/Snf both in vivo and in vitro (Roberts and Winston 1997; Hassan et al. 2002, 2006; Geng and Laurent 2004; Chandy et al. 2006; Chatteriee et al. 2011). To analyze binding of Swi/Snf to acetylated nucleosomes, 25N47 nucleosomes were reconstituted as before and acetylated using purified Ada2-Tap complex containing the acetyltransferase Gcn5. Binding to acetylated nucleosomes was assayed as a function of increasing Swi/Snf concentration with a fixed concentration of nucleosomes (Fig. 3B). As a control, binding to unacetylated nucleosomes was also studied (Fig. 3A). Interestingly, Swi/Snf complexes with no acetylation on Snf2 showed greater binding to acetylated nucleosomes compared with the wild type (Fig. 3B).

Swi/Snf functions to remodel nucleosomes by sliding or evicting histones (Gutierrez et al. 2007). To test whether the difference in binding resulted in altered sliding activity, we carried out remodeling reactions using nucleosomes reconstituted on 601-containing DNA with a 72-bp linker (N72), which was acetylated as described earlier. Remodeling was assayed as a function of both time (Supplemental Fig. 4a,b) and Swi/Snf concentration (Fig. 3C,D). As observed in the case of binding, Swi/Snf complexes with unacetylated Snf2 displayed greater efficiency in sliding acetylated nucleosomes compared with the wild type (Fig. 3D; Supplemental Fig. 4b). No significant difference in sliding activity was observed on unacetylated nucleosomes (Fig. 3C; Supplemental Fig. 4a). Since our previous binding experiments were carried out on $25 \mathrm{~N} 47$ nucleosomes, we tested binding of Swi/Snf complexes to acetylated N72 mononucleosomes, which showed results similar to those on 25N47 nucleosomes (Supplemental Fig. 5b). Since remodeling reactions were carried out in the presence of ATP, we tested binding under similar conditions. Interestingly, addition of ATP increased binding of Swi/ Snf complexes to nucleosomes, but, as shown earlier, differences in binding between the wild type and the mutant were observed only on acetylated nucleosomes (Supplemental Figs. 5b, 6).

AT-hook domains in Snf2 are adjacent to the ATPase/ helicase domain. To test whether acetylation of Snf2 could influence the ATPase activity of Swi/Snf, hydrolysis of ATP was monitored in the presence of DNA or nucleosomes (unacetylated and acetylated). To rule out changes in ATPase activity arising from differential affinities for nucleosomal substrates, all ATPase assays were performed in the presence of excess DNA or nucleosomes. No difference in ATPase activity was observed (Supplemental Fig. 4c). Thus, acetylation of Snf2 specifically regulates binding to acetylated nucleosomes and restricts remodeling of these nucleosomes by Swi/Snf without altering the ATPase activity.

\section{Levels of histone acetylation at genes regulate differential occupancy of Swi/Snf}

Based on in vitro results, levels of histone acetylation at genes would be expected to affect Swi/Snf occupancy. Acetylation of histones by SAGA has been shown to increase Swi/Snf occupancy at genes (Roberts and Winston 1997; Hassan et al. 2002, 2006; Geng and Laurent 2004; Chandy et al. 2006; Chatterjee et al. 2011). We carried out ChIP-seq experiments using H3K9Ac antibody and analyzed acetylation levels at genes in groups 1,2, and 3 as classified earlier. Group 1 genes showed moderate levels of histone acetylation during normal growth conditions (Fig. 4A), which was significantly reduced upon stress (Fig. 4B,C). This would be expected for genes that are down-regulated by stress. Group 3 genes also displayed moderate levels of acetylation, with no significant change during stress (Fig. 4G-I). The greatest changes in histone acetylation were observed in the case of group 2 genes, which showed low levels of histone acetylation during normal growth conditions (Fig. 4D) but were significantly enriched for histone acetylation upon induction of stress (Fig. 4E,F). Accordingly, this is the same set of genes in which we observed greater differences in Snf2 binding in the 2R mutant compared with the wild type. We further hierarchically clustered genes based on Snf2 and H3K9Ac occupancies. Separate clustering was done for unstressed and stressed conditions (Supplemental Fig. 7a). We found that increased acetylation correlated with the changes in Snf2 occupancy observed for the $2 \mathrm{R}$ mutant. These results are in line with our in vitro experiments and show that differences in occupancy result from altered affinities for acetylated nucleosomes depending on the acetylation status of Snf2.

Since our observations suggest that acetylation of Snf2 plays an important role in mediating Swi/Snf occupancy at genes during stress, we next asked what effect stress has on regulating acetylation of Snf2. Swi/Snf complexes were purified from wild-type strain grown under conditions similar to those used in ChIP-seq experiments. Using antiacetylated lysine antibody to probe for Snf2 acetylation and anti-Snf2 antibody as loading control, we found an $\sim 1.3$-fold increase in acetylation of Snf2 purified from cells that were stressed (Supplemental Fig. 7b), suggesting that most of Snf2 is acetylated during stress. This increased acetylation would not only help release of Swi/Snf from genes that are repressed by stress but also regulate occupancy at genes that are up-regulated during stress conditions.

Our previous study showed that the purified bromodomain of Snf2 (amino acids 1547-1703) could bind the purified AT-hook domain (amino acids 1384-1546) when both lysine residues between the AT hooks were acety- 
A
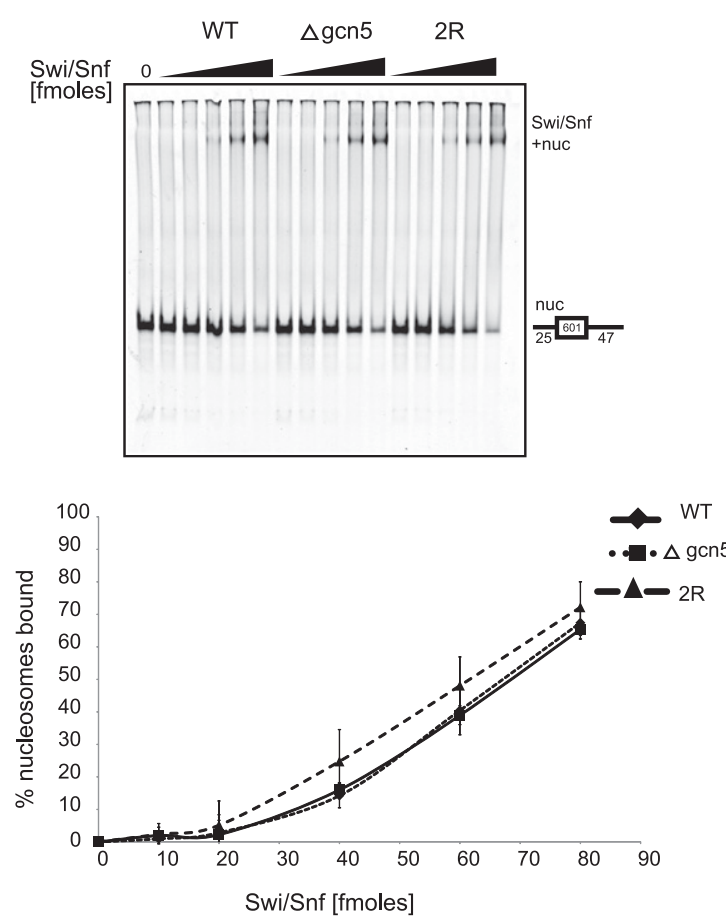

C
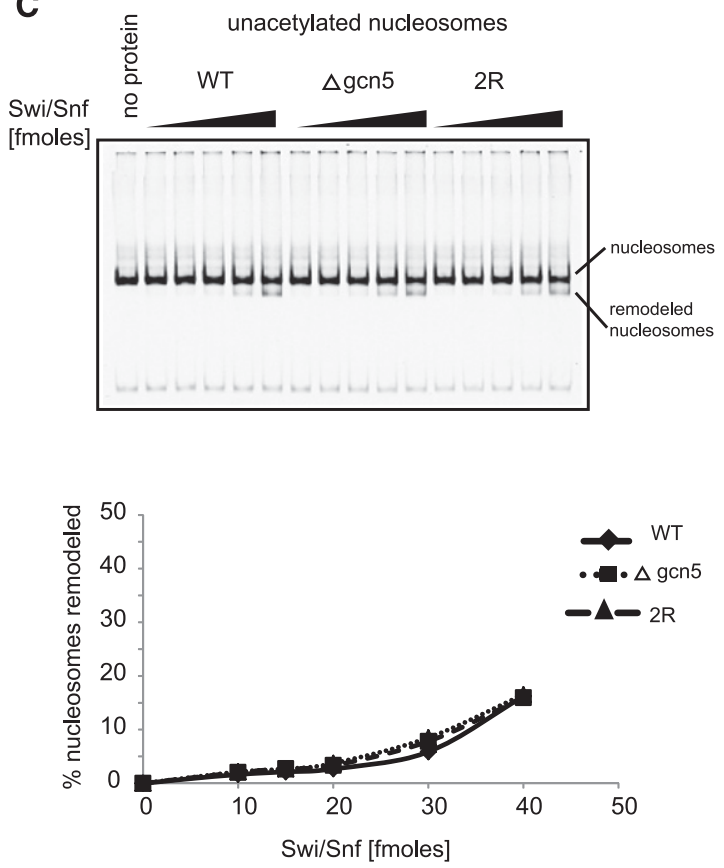

B
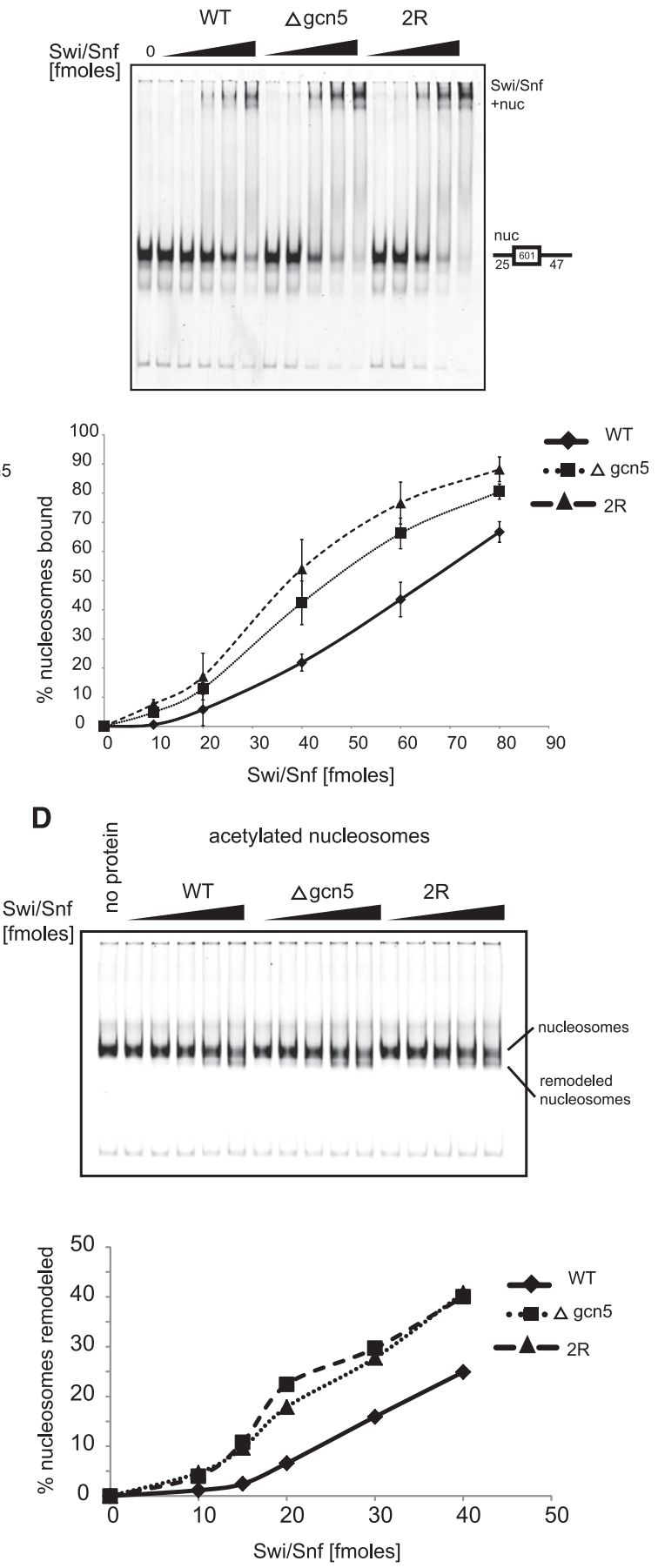

Figure 3. Snf2 acetylation regulates Swi/Snf binding and remodeling of acetylated nucleosomes. Twenty femtomoles of $5^{\prime}$ Cy5-labeled unacetylated 25N47 mononucleosomes $(A)$ and $20 \mathrm{fmol}$ of 5' Cy5-labeled acetylated 25N47 mononucleosomes $(B)$ were incubated with increasing concentrations of Swi/Snf complexes $(0,10,20,40,60$, and $80 \mathrm{fmol})$ and analyzed by native PAGE. Bands indicating unbound mononucleosome and Swi/Snf-bound mononucleosomes are indicated. Plots for Swi/Snf binding represent mean values \pm SEM for at least three independent experiments. Remodeling of unacetylated $(C)$ versus acetylated $(D)$ nucleosomes as a function of Swi/Snf concentration. In each case, $10 \mathrm{fmol}$ of mononucleosomes was incubated with increasing concentrations of Swi/Snf complexes $(0,10,15,20,30$, and $40 \mathrm{fmol})$ in the presence of $1.5 \mathrm{ng}$ of competitor salmon sperm DNA. Reactions were carried out for $30 \mathrm{~min}$. Plots for remodeling are shown.

lated in vitro by Gen5 (Kim et al. 2010). This intramolecular interaction suggests that acetylated $\mathrm{H} 3$ histone tails on nucleosomes would compete with acetylated lysine residues in Snf2 for binding to the bromodomain. Thus, Snf2 acetylation could restrict binding of Swi/ Snf to nucleosomes with low levels of acetylation. We 
Dutta et al.
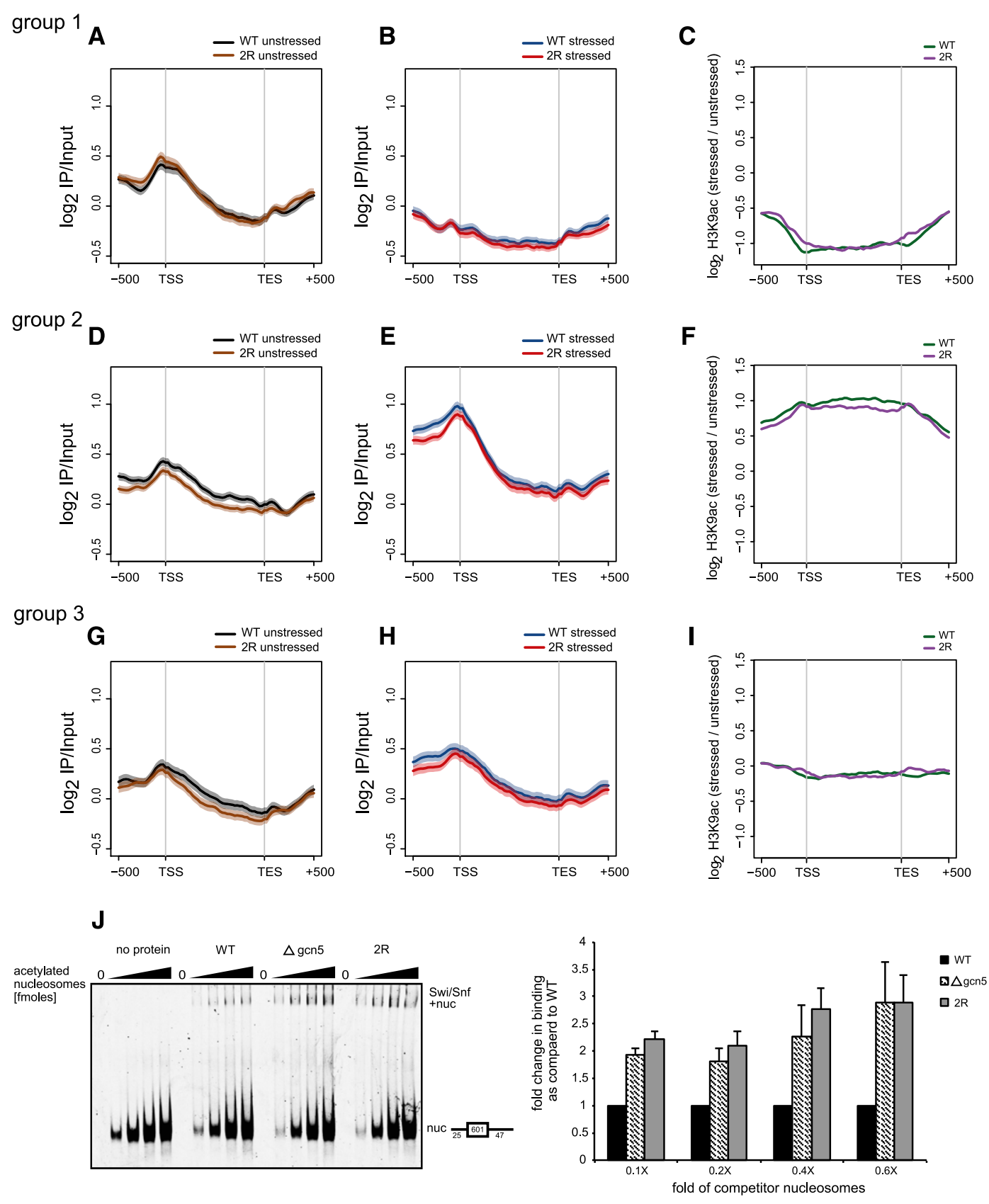

Figure 4. Histone acetylation levels determine differential recruitment of Swi/Snf between the wild type (WT) and mutant. Histone acetylation was assayed by ChIP experiments using H3K9Ac antibody in the wild-type and Snf2 K1493R K1497R (2R) strains. Log 2 ratios of normalized enrichment (immunoprecipitation [IP]/input) for each gene region, including 500 bp upstream of and downstream from the gene, were used for average gene analysis. The TSS and TES are indicated. Transparent shading around the line indicates the standard error of the mean. Genes were grouped based on changes in Snf2 occupancy as described earlier. H3K9Ac at group 1 genes in unstressed $(A)$ and stressed $(B)$ conditions, group 2 genes in unstressed $(D)$ and stressed $(E)$ conditions, and group 3 genes in unstressed $(G)$ and stressed $(H)$ conditions are shown. $(C, F, I)$ Average $\log _{2}$ ratios of changes in H3K9Ac occupancy during stressed conditions for each gene region, including $500 \mathrm{bp}$ upstream of and downstream from the gene, were calculated and plotted for both the wild type and Snf2 K1493R K1497R (2R) for each group. (J) Competition assay was carried out by binding $25 \mathrm{fmol}$ of Swi/Snf complexes (purified from wild-type, Snf2 K1493R K1497R [2R], and $\Delta$ gcn5 strains) to $100 \mathrm{fmol}$ of unlabeled unacetylated $25 \mathrm{~N} 47$ nucleosomes followed by competition with increasing concentrations $(0,10,20,40$, and $60 \mathrm{fmol})$ of acetylated 5' Cy5-labeled 25N47 nucleosomes. Swi/Snfbound complexes were resolved by native PAGE. Bands indicating unbound mononucleosomes and Swi/Snf-bound mononucleosomes are indicated. Plots for Swi/Snf binding are normalized to wild-type Swi/Snf binding. 
carried out competition experiments in vitro in which Swi/Snf was prebound to excess unacetylated $25 \mathrm{~N} 47$ nucleosomes with unlabeled DNA followed by competition with increasing concentration of acetylated 25N47 nucleosomes with 5' Cy5-labeled DNA. Addition of increasing concentrations of acetylated nucleosomes caused the mutant Swi/Snf complexes to be released more readily from unacetylated nucleosomes, allowing twofold or more binding to acetylated nucleosomes as compared with the wild type (Fig. 4J). As a control, competition was carried out with increasing concentrations of unacetylated 25N47 nucleosomes with 5' Cy5 DNA label. No differences in binding were observed between the wild type and mutants in this case (Supplemental Fig. 7c). Thus, interaction of the bromodomain with acetylated lysine residues in Snf2 affects its binding to acetylated histones by reducing the overall affinity of the Swi/Snf complex for acetylated nucleosomes. The presence of high levels of histone acetylation in genes, as in group 2, would be needed to compete for binding to the bromodomain of Snf2, thus stabilizing occupancy of Swi/ Snf at promoters of these genes.

\section{Snf2 acetylation regulates nucleosome occupancy during stress}

Loss of Snf2 acetylation regulates Swi/Snf occupancy during stress, and our in vitro experiments show that it leads to increased remodeling by the Swi/Snf complex on acetylated nucleosomes. To observe the effects that acetylation of Snf2 has in vivo, we analyzed nucleosome organization during growth in unstressed and stressed conditions by micrococcal nuclease (MNase) digestion followed by paired-end sequencing. Genes were grouped as in Figure 1B, and average nucleosome plots around the TSS were generated. No differences in nucleosome occupancies were observed in group 1 genes during unstressed conditions (Fig. 5A). However, while induction of stress led to an increase in nucleosome occupancy at the NFR and over the TSS in the wild type, the $2 \mathrm{R}$ mutant showed less nucleosome occupancy at these positions with no changes, compared with the wild type, observed over the body of the gene (Fig. 5B). These changes in nucleosome occupancy in the $2 \mathrm{R}$ mutant are most likely due to the slow release of Snf2 observed in this mutant (Fig. 2B). The group 1 genes RPS26b and HXT3 lose Snf2 occupancy during stress, but the $2 \mathrm{R}$ mutant is slower to release from these genes compared with the wild type. Consequentially, we also observed lower nucleosome occupancy around the promoter of these genes (Supplemental Fig. 8a,b). Induction of stress reduced nucleosome occupancy for both group 2 and group 3 genes (Fig. 5C-F). Furthermore, consistent with the increased Snf2 occupancy in the $2 \mathrm{R}$ mutant in group 2 genes during stress (Fig. 2E,F), we found a significant decrease in nucleosome occupancy at the NFR compared with the wild type (Fig. 5D). Interestingly, among group 2 genes, YGR043C, which has a greater occupancy of Snf2 in $2 \mathrm{R}$ compared with the wild type during stress, shows reduced nucleosome occupancy (Supplemental Fig. 8d). On the other hand, genes with a similar occupancy of Snf2 in the wild type and 2R during stress (e.g., HXK1) did not show changes in nucleosome occupancy (Supplemental Fig. 8c). The increased occupancy of Snf2 observed in 2R for group 3 genes (Fig. 2H) also affects nucleosome occupancy around the TSS (Fig. 5F). In accordance with no significant change observed in Snf2 occupancy between the wild type and $2 \mathrm{R}$ during unstressed conditions, we did not observe significant changes in nucleosome positioning for these groups of genes. Thus, acetylation of Snf2 and its increase during stress can regulate nucleosome occupancy specifically at promoters of stress-responsive genes by modulating the residency of the Swi/Snf complex.

\section{Dynamics of Swi/Snf recruitment and release} from genes are regulated by acetylation of Snf2

Repression of group 1 genes showed a defect in the release of Swi/Snf from promoters in the 2R mutant (Fig. 2B). On further analysis of this group, we identified a subset of genes (Supplemental Table 2) in which both the wild type and 2R mutant showed similar levels of Swi/Snf occupancy under the unstressed condition (Fig. 6A). However, upon induction of stress, while the wild type showed no occupancy of Swi/Snf, the 2R mutant remained occupied at the promoter (Fig. 6B). GO term analysis of this group showed that it was enriched for genes involved in ribosome synthesis and translation, biological processes that are suppressed during stress in yeast (Shivaswamy and Iyer 2008). Analysis of histone acetylation levels showed that although these genes are turned off during stress, low levels of acetylation persisted at these genes (Supplemental Fig. 9a,b). Also, analysis of MNase sequencing (MNase-seq) data showed lower nucleosome occupancy around the TSS in the 2R mutant compared with the wild type during stress (Fig. 6C). Transcriptional repression of genes would cause wild-type Swi/Snf with high levels of Snf2 acetylation to have less affinity for nucleosomes with reduced acetylation and would be more readily lost as compared with the mutant.

It had been shown earlier that at some stress-responsive genes, Snf2 occupancy in a $\Delta \mathrm{gcn} 5$ strain is increased compared with the wild type under normal growth conditions (Geng and Laurent 2004; Govind et al. 2005; Zhang et al. 2008). Since the $2 \mathrm{R}$ mutant would mimic loss of acetylation of Snf2, as in the case of $\Delta g c n 5$, we asked whether differential recruitment of Swi/Snf was observed in group 2 genes during unstressed conditions. Interestingly, the 2R mutant was preoccupied at the promoters of a subset of genes under unstressed conditions where no occupancy of wild-type Swi/Snf was observed (Fig. 6D,E; Supplemental Table 2). This list was enriched for genes involved in response to stress, $\mathrm{NAD} / \mathrm{NADH}$ metabolism, and aging in yeast. Analysis of histone acetylation levels in these genes showed that during unstressed conditions, these genes are associated with significant levels of histone acetylation (Supplemental Fig. 9c,d) that would allow unacetylated Snf2 in 2R to bind while occluding the wild-type Swi/Snf complex. Interestingly, some increased nucleosome occupancy over the ORF was observed for 
Dutta et al.

\section{group 1}

A

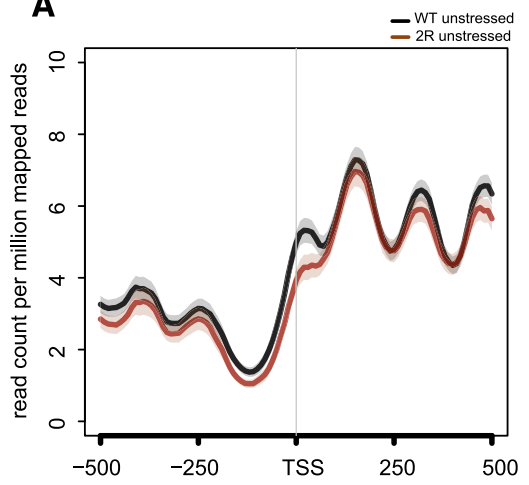

group 2

C

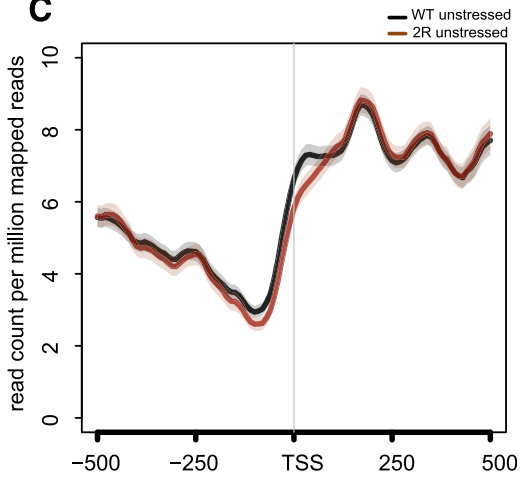

group 3

E

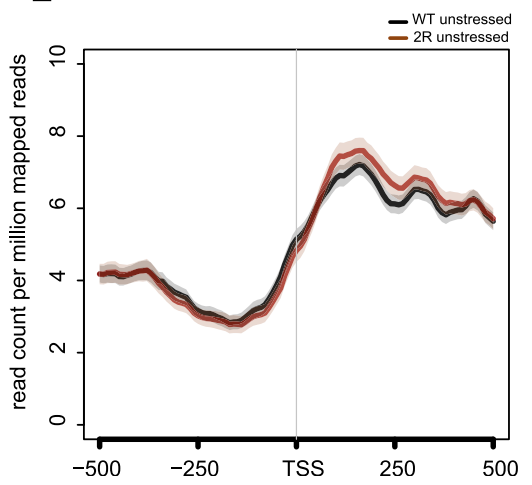

B
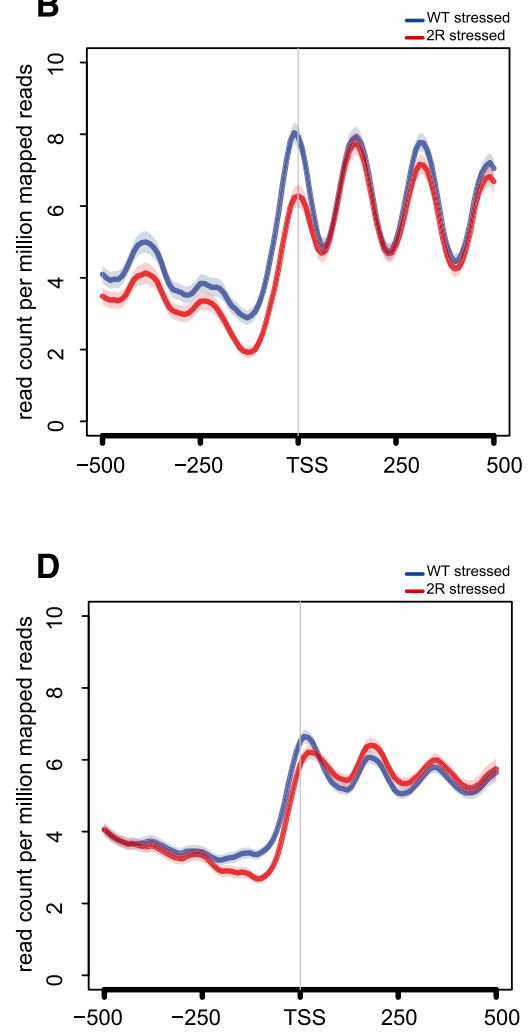

$\mathbf{F}$

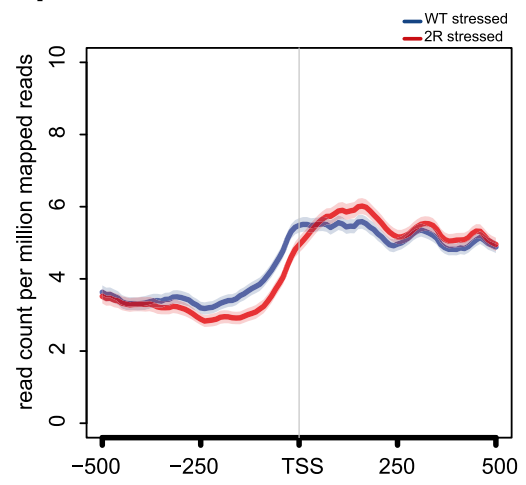

Figure 5. Differential remodeling due to Snf2 acetylation affects nucleosome occupancy in genes. Nucleosome positioning was determined by MNase-seq. The average nucleosome profile in wild-type (WT) and Snf2 K1493R K1497R (2R) strains for group $1(A, B)$, group $2(C, D)$, and group $3(E, F)$ genes during unstressed and stressed conditions was calculated and plotted $500 \mathrm{bp}$ upstream of and downstream from the TSS using ngsplot. Transparent shading around the line indicates the standard error of the mean. the $2 \mathrm{R}$ mutant in these genes during unstressed condition (Fig. 6F; Supplemental Fig. 8e,f). Our analysis of genomewide data suggests that acetylation may function to finetune levels of Swi/Snf in genes and further prevent aberrant engagement at the promoters of genes.

\section{Loss of the Snf2 bromodomain regulates Swi/Snf occupancy during stress}

Our studies thus far suggest that in the $2 \mathrm{R}$ mutant, loss of competition for acetylated lysine residues in Snf2 allows acetylated histones to bind the Snf2 bromodomain more readily. To test whether differences in Snf2 occupancy observed between the wild type and $2 \mathrm{R}$ were indeed reliant on the bromodomain, we carried out ChIP-seq analysis for
Snf2 occupancy under conditions described earlier using strains lacking the Snf2 bromodomain (Snf2 1-1547) in both the wild type and $2 \mathrm{R}$. Our previous analysis was carried out using an antibody that targeted the $\mathrm{C}$ terminus of Snf2. Since loss of the bromodomain could affect interaction with this antibody, we used antibodies that target the $\mathrm{N}$ terminus of Snf2. For consistency of our results and interpretation of the data, we repeated the ChIP-seq analysis using this antibody for both the wild-type and 2R strains. We grouped genes as in Figure 1C. On comparing Snf2 occupancy in the wild type and $\Delta$ bromodomain mutant (wild-type $\Delta$ bromo), we observed reduction in Snf2 occupancy in wild-type $\Delta$ bromo specifically during stress (Fig. 7A). Also, loss of the bromodomain resulted in a greater loss of Snf2 in group 1 genes during stress. Interestingly, these results are the 
A

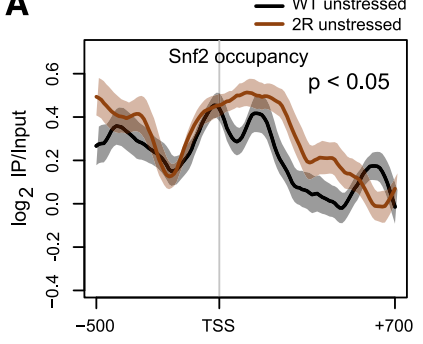

B

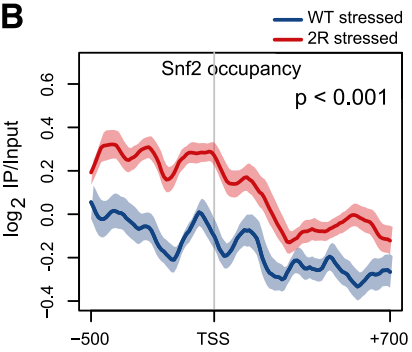

D

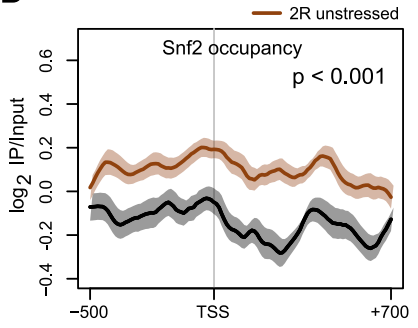

E

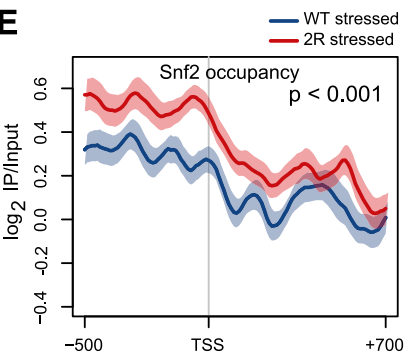

C

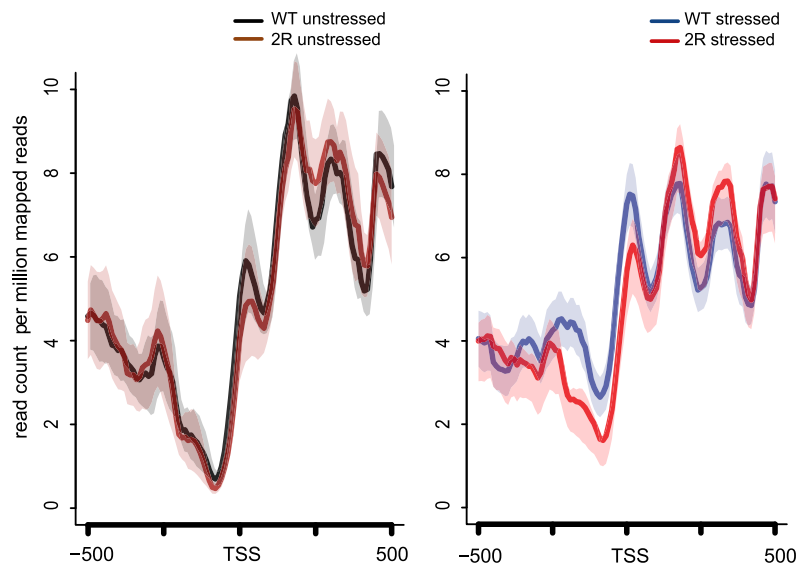

F

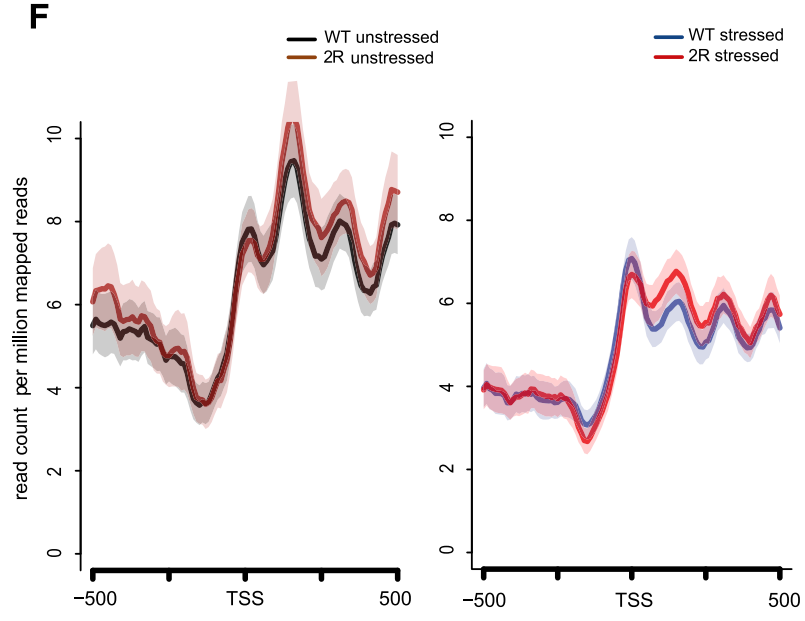

Figure 6. Acetylation of Snf 2 prevents aberrant engagement of Swi/Snf in stress-dependent genes. Snf 2 occupancy $(A, B)$ and nucleosome occupancy $(C)$ in genes in which Swi/Snf is slow to release from the promoters and ORF in Snf2 K1493R K1497R (2R) compared with the wild type (WT). This set of genes was defined as a subset of group 1 in which the sum of normalized $\log _{2}$ immunoprecipitation (IP)/input TSS $(-700 /+500)$ coverage values was $<0$ in the wild type (stressed) and $>0$ in $2 \mathrm{R}$ (stressed) and was plotted. Snf2 occupancy $(D, E)$ and nucleosome occupancy $(F)$ in genes in which Swi/Snf is preoccupied at the promoters and ORF in Snf2 K1493R K1497R (2R) but not in the wild type. Genes in group 2 in which the sum of normalized $\log _{2}$ immunoprecipitation/input TSS $(-700 /+500)$ coverage values was $<0$ in wild type (unstressed) and $>0$ in $2 \mathrm{R}$ (unstressed) were used for the genome average plot. Transparent shading around the line indicates the standard error of the mean. $P$-values are based on mean values per gene in the TSS region $(-700 /+500)$ and are from a two-sided Student's $t$-test between samples.

opposite of those observed for the 2R mutant (Figs. 1, 2). Furthermore, no significant change in occupancy was observed for group 1 and group 3 genes in the unstressed condition. These results suggest that the increased occupancy of Swi/Snf observed due to loss of Snf2 acetylation was indeed dependent on bromodomain interactions. We found that levels of histone acetylation regulated changes in Snf2 occupancy observed for the 2R mutant. We therefore clustered group 2 genes into four quartiles arranged on the basis of highest to lowest levels of H3K9Ac in the wild type during stress (Fig. 7B) and carried out difference analysis of Snf2 occupancy between the wild-type and mutant strains. In agreement with the competition model for binding the Snf2 bromodomain, genes with the highest H3K9Ac levels (first quartile) have increased Snf2 occupancy in $2 \mathrm{R}$ compared with the wild type along with having the lowest Snf2 occupancy in the bromodomain deletion strains. Conversely, genes with the lowest levels of H3K9Ac show no significant changes in Snf2 occupancy. These results suggest that competition between Snf2 acetylated residues and 
Dutta et al.

A

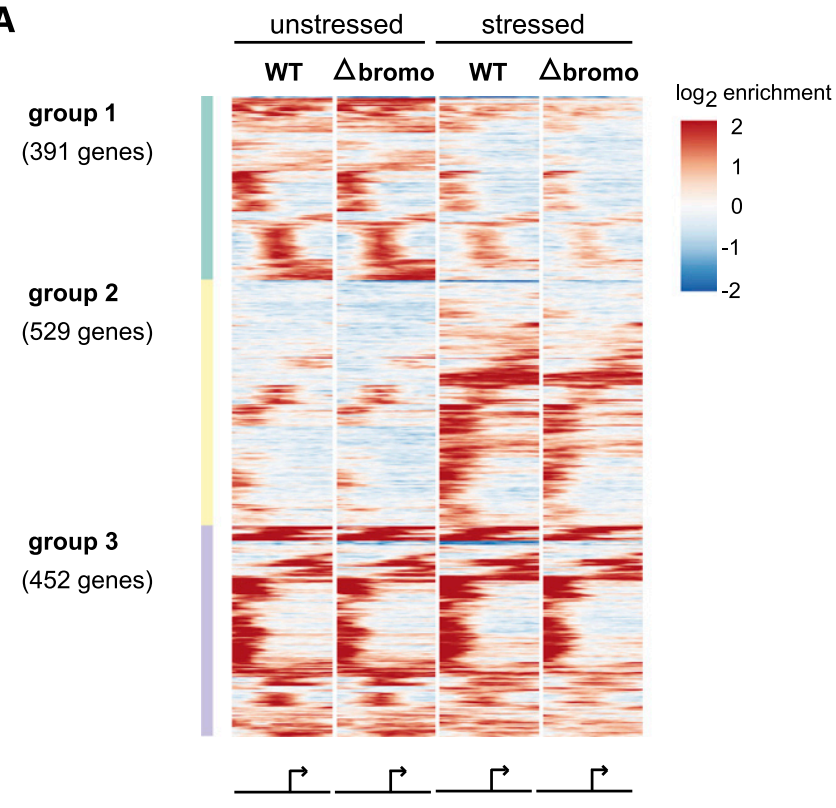

B

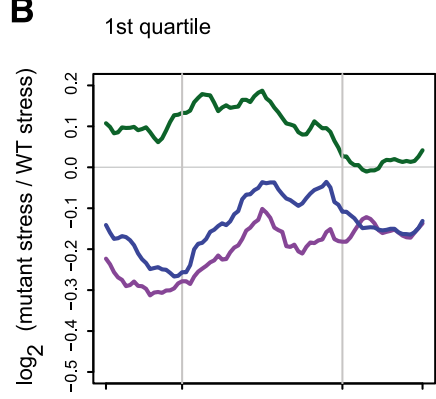

2nd quartile

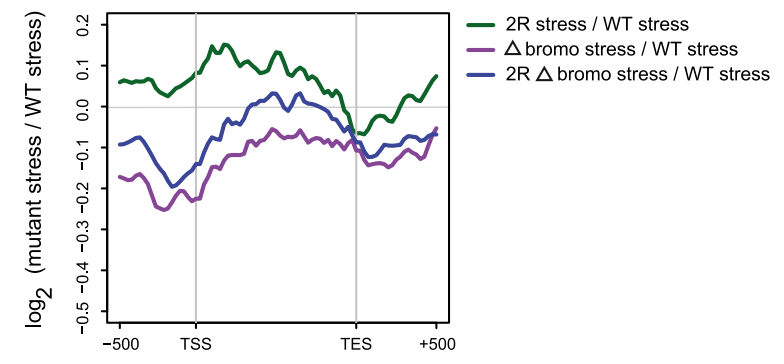

3rd quartile

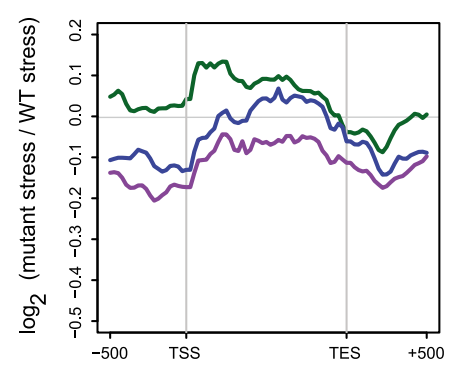

4th quartile

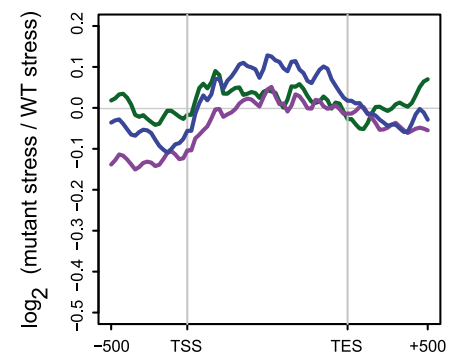

Figure 7. Bromodomain of Snf2 regulates Swi/Snf occupancy during stress. (A) Genes were grouped based on changes in Snf2 occupancy during stress as in Figure 1C. The heat map of Snf2 occupancy for the wild type (WT) and the Snf2 $\Delta$ bromodomain in each group under stressed and unstressed conditions was generated by hierarchically row-clustering normalized $\log _{2}$ (immunoprecipitation [IP]/input) values for regions $-700 /+500$ around the TSS of genes. (B) Group 2 genes were clustered based on the highest to lowest levels of H3K9Ac and divided into quartiles. Average gene analysis was done as described in the Materials and Methods. Difference profiles for changes in Snf2 occupancy in mutants compared with the wild type for each quartile are shown. The TSS and TES are indicated.

acetylated histones for binding the Snf2 bromodomain is key to regulating Swi/Snf occupancy at genes during stress.

Activator increases retention of the Swi/Snf complex, thus mitigating lower affinity for nucleosomes due to Snf2 acetylation

The differences in Snf2 occupancy in group 2 genes that are up-regulated by stress can be explained as resulting from increased levels of histone acetylation in these genes. Based on our in vitro results, it would be expected that lack of Snf2 acetylation of the 2R mutant would allow its bromodomain to bind acetylated histone tails with higher affinity in the absence of competition when compared with the wild type. However, genes in groups 1 and 3 that have similar levels of Swi/Snf occupancy in the wild type and $2 \mathrm{R}$ contain histone acetylation marks, 
albeit at moderate levels. Thus, histone acetylation levels alone do not explain why significant differences in Snf2 occupancy were not observed at genes in groups 1 and 3 . Transcriptional activators have been shown to play an important role in helping recruit the Swi/Snf complex along with coactivators such as SAGA /Chandy et al. 2006; Ferreira et al. 2009). Additionally, in vitro, a chimeric construct of VP16 activator fused to a Gal4 DNAbinding domain has been shown to target Swi/Snf specifically to activator-bound nucleosomes (Prochasson et al. 2003; Gutierrez et al. 2007). We therefore tested what role Snf2 acetylation played in differential targeting of Swi/ Snf complexes in the presence of activator. Binding of Swi/Snf was tested on unacetylated/acetylated N72 nucleosomes with Gal4-binding sites in the adjacent linker DNA. Nucleosomes were preincubated with Gal4-VP16 or buffer only to allow binding of Gal4-VP16 to DNA. To observe specific targeting of Swi/Snf to activator-bound nucleosomes, all binding reactions were carried out in the presence of excess competitor DNA (Gutierrez et al. 2007). Under these reaction conditions, wild-type and mutant complexes bound unacetylated nucleosomes very weakly, and binding was stimulated to similar levels in the presence of Gal4-VP16 (Fig. 8A). Both wild-type and mutant Swi/Snf bound acetylated nucleosomes better than unacetylated nucleosomes, with mutants showing increased affinity for acetylated nucleosomes, as shown before (Fig. 8B). Interestingly, in the presence of activator, all complexes bound acetylated nucleosomes equally well (Fig. 8B). Likewise, remodeling of nucleosomes by wildtype and mutant complexes showed similar results, as observed for binding in the presence of activator (Fig. 8C, $\mathrm{D}$ ). This result is surprising, as it suggests that activatordependent recruitment would play a role in compensating for the reduced binding of Swi/Snf to genomic loci with low to moderate levels of histone acetylation.

Our in vitro results would predict that in the presence of activator, bromodomain-dependent interactions would play less significant roles in Snf2 occupancy. This would allow for similar recruitment of Snf2 irrespective of its acetylation status or the presence of the Snf2 bromodomain. Also, the presence of activator increases recruitment of Swi/Snf even in the presence of histone acetylation. This would suggest that genes with the highest levels of Snf2 occupancy would most likely be more dependent on activators. Among genes in group 2, HXT6 and HXT7, which encode for high-affinity glucose transporters under growth in glucose-depletion conditions, are bound by the activator Adr1 and show high but similar occupancy of wild-type and mutant Snf2 at their promoters (Fig. 8E; De Winde et al. 1996; Tachibana et al. 2005). Similar observations were seen at MET13, a Gcn4-regulated gene (Supplemental Fig. 10b). On the other hand, YPL270W, encoding for a mitochondrial inner membrane half-type $\mathrm{ABC}$ transporter but not found to be regulated by an activator, shows less occupancy of Snf2 and greater differences between the wild type and mutants (Fig. 8E). YPL225W and UIP4 showed similar changes in Snf2 occupancy between the wild type and mutants as well (Supplemental Fig. 10c,d).
Small differences in Swi/Snf occupancy between the wild type and $2 \mathrm{R}$ were observed for groups 1 and 3 genes during growth in the unstressed condition. These groups encode for genes that regulate translation and metabolic processes that are critical for cell viability (Supplemental Fig. 1). These genes are most likely dependent on activators to maintain Swi/Snf occupancy to keep these genes in an ON state. Indeed, group 1 genes such as RPL31B, a ribosomal protein gene, and group 3 genes such as $R P N 4$, a transcription factor that stimulates expression of proteasome genes, showed similar occupancy of Snf2 (Supplemental Fig. 10a,e).

\section{Discussion}

Investigating the role of Snf2 acetylation on functions of Swi/Snf, we found that genes that are induced upon stress caused by nutrient deprivation showed a greater recruitment of Swi/Snf in the absence of Snf2 acetylation (Fig. 2E). Also, a subset of these genes showed prestress occupancy of Snf2 only in the 2R mutant during normal growth conditions (Fig. 6D,E). Our observations help explain previous studies in which increased occupancy of Snf2 was observed in a $\Delta g c n 5$ strain in the stressinducible genes RNR3, SUC2, and ARG1 specifically in the absence of stress (Geng and Laurent 2004; Govind et al. 2005; Zhang et al. 2008). Biochemical analysis supported our in vivo observations in which complexes purified from the $2 \mathrm{R}$ and $\Delta g c n 5$ strains bound and remodeled acetylated nucleosomes better than the wild type, while no differences were observed in unmodified nucleosomes (Fig. 3). Stress-inducible genes need to be quickly turned on and turned off as a response to environmental stimuli. This would necessitate a quick turnover of chromatin remodelers to open and close promoters of stress-dependent transcription. Turning on these genes is associated with an increase in levels of H3K9 acetylation coupled with an increase in acetylation of Snf2 (Fig. 4E; Supplemental Fig. 7b). High levels of histone acetylation are needed to outcompete acetylation of Snf2, allowing for interaction with the bromodomain and recruitment of the Swi/Snf complex. This allows transcription at these genes to be tightly regulated. Furthermore, the presence of activator at these genes can also stabilize Swi/Snf occupancy. In agreement with our in vitro results that show increased recruitment of Swi/Snf to acetylated nucleosomes in the presence of activator (Fig. 8B), we found that the genes showing the highest occupancy of Snf2 are not affected by loss of acetylation or the bromodomain of Snf2. However, genes with moderate to low levels of Swi/ Snf recruitment showed increased dependence on Snf2 bromodomain interactions (Fig. 8E; Supplemental Fig 10c,d). Thus, recruitment and functioning of Swi/Snf at these genes may be more dependent on histone acetylation. During unstressed conditions, the absence of activator coupled with intramolecular interaction of the bromodomain with acetylated lysine residues in Snf2 would occlude binding to lowly acetylated histone tails at the promoters of these genes. Thus, acetylation of Snf 2 can play important roles by helping direct cellular resources 
A

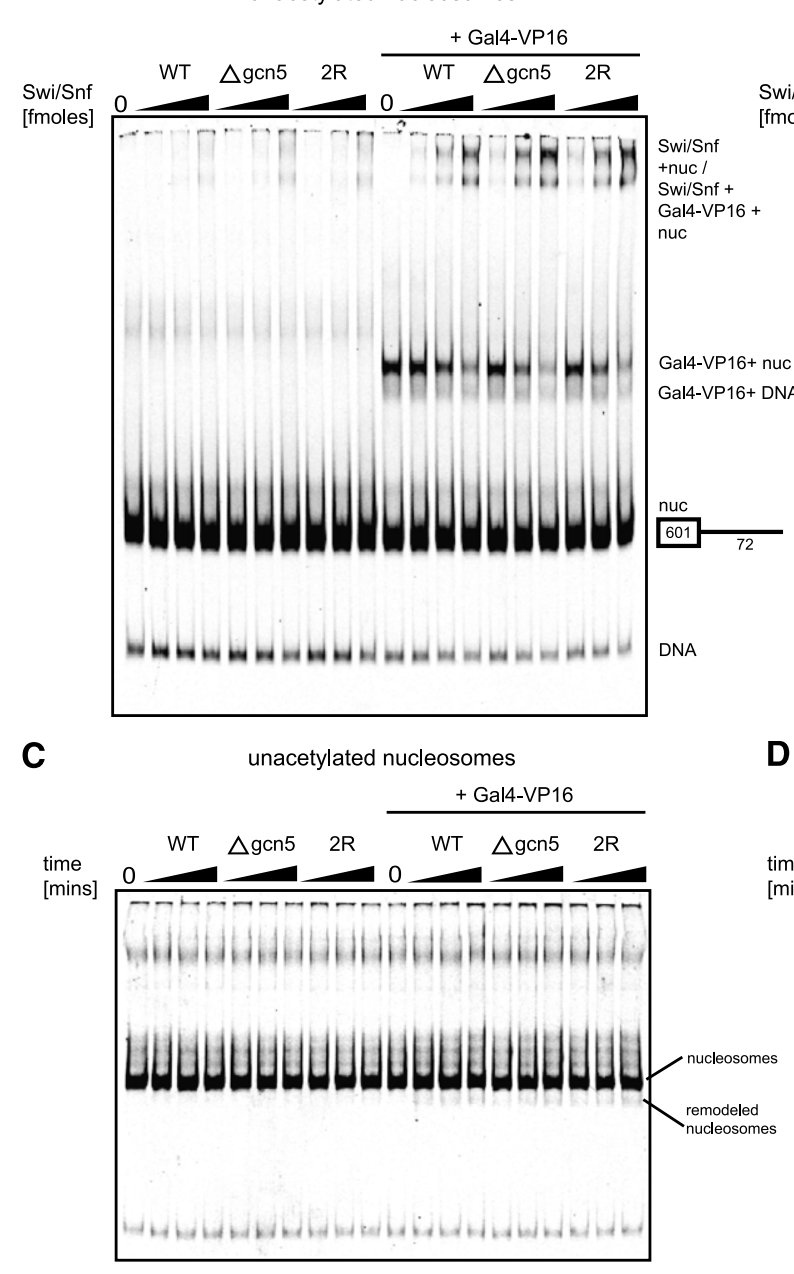

E

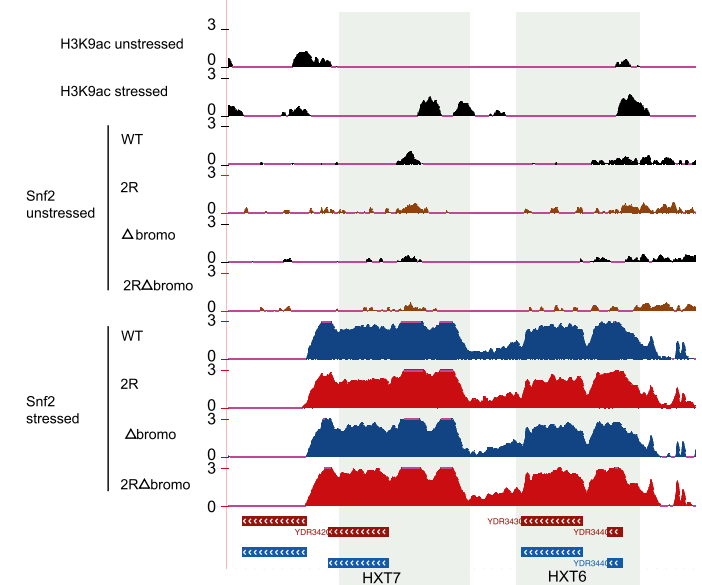

B

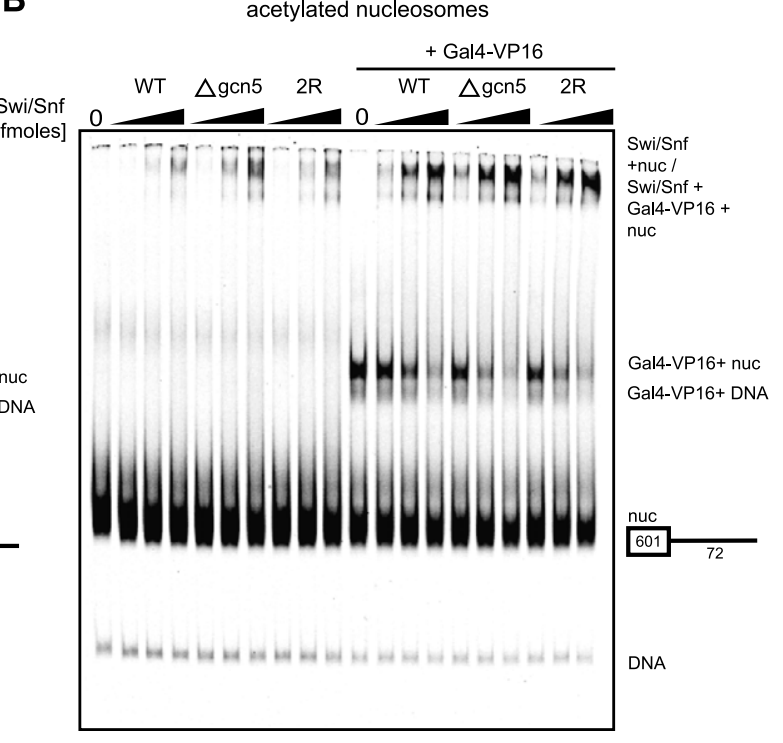

D
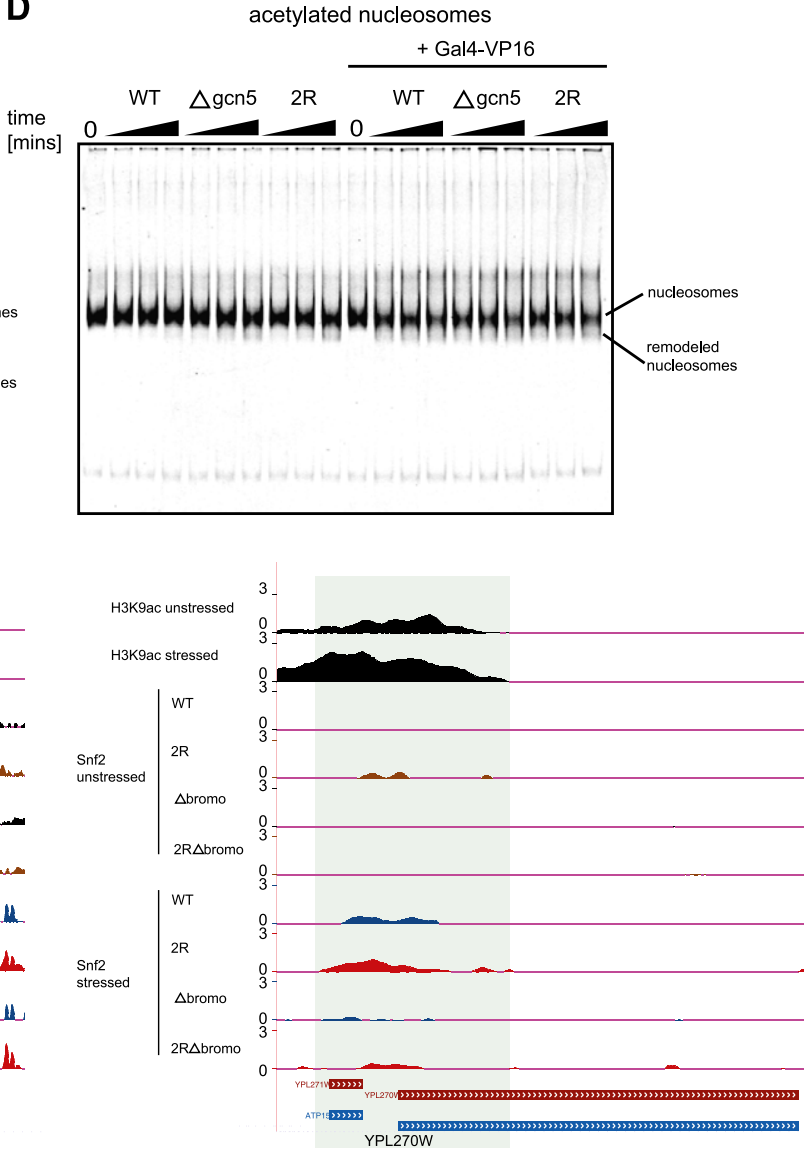

Figure 8. The presence of activator increases binding and remodeling of the wild-type (WT) Swi/Snf complex to levels similar to that of the mutant with unacetylated Snf2. To assay for binding in the presence of Gal4-VP16, 20 fmol of 5' Cy5-labeled unacetylated N72 mononucleosomes $(A)$ or $20 \mathrm{fmol}$ of $5^{\prime}$ Cy5-labeled acetylated N72 mononucleosomes $(B)$ containing Gal4-binding sequence was incubated with/without $200 \mathrm{fmol}$ of Gal4-VP16 followed by binding with increasing concentrations of Swi/Snf complexes $(0,20$, 40, and $60 \mathrm{fmol}$ ) in the presence of $2 \mathrm{ng}$ of competitor salmon sperm DNA and was analyzed by native PAGE. Bands indicating unbound mononucleosome, Gal4-VP16-bound DNA/mononucleosome, and Swi/Snf-bound mononucleosomes or Swi/Snf-bound Gal4-VP16 + mononucleosome are indicated. Remodeling of Gal4-VP16-bound unacetylated $(C)$ or acetylated $(D)$ mononucleosomes was assayed using $20 \mathrm{fmol}$ of 5' Cy5-labeled mononucleosomes with $20 \mathrm{fmol}$ of Swi/Snf complex (from wild-type, Snf2 K1493R K1497R [2R], and $\Delta$ gcn5 strains) and $200 \mathrm{fmol}$ of Gal4-VP16 in the presence of $2 \mathrm{ng}$ of competitor salmon sperm DNA as a function of time $(5,15$, and $30 \mathrm{~min}) .(E)$ Genome browser screenshots of Snf2 occupancy for wild type, 2R, Snf2 $\Delta$ bromodomain, and 2R $\Delta$ bromodomain strains at HXT6 and HXT7 that are regulated by Adr1 and YPL270W that were not found to be bound by activator using online tool YEASTRACT. The top two rows in each genome browser screenshot show wild-type H3K9Ac occupancy during unstressed and stressed conditions. 
toward cell survival. It can quickly down-regulate some processes by helping remove Swi/Snf from promoters, which increases promoter nucleosome occupancy (Fig. $5 \mathrm{~B}$ ), causing genes to reduce transcriptional output while at the same time up-regulating genes that are important for adaptation and survival during stress.

Genes occupied by Swi/Snf during unstressed conditions (group 1) or under both unstressed and stressed conditions (group 3) showed no significant differences in occupancy between wild type, $2 \mathrm{R}$, or bromodomain deletion mutants (Figs. 1, 2). This was surprising because these genes were associated with moderate levels of histone acetylation marks (Fig. 4), a condition under which the 2R mutant without acetylable lysine residues should bind better than the wild type, and Snf2 without the bromodomain would be expected to bind more weakly. In trying to understand why this might be the case, we found that the presence of activators stimulated the binding and remodeling activities of Swi/Snf to similar levels irrespective of the acetylation statues of Snf2 (Fig. 8). Previous studies have highlighted the role of activators in regulating the recruitment and retention of the Swi/Snf complex both in vivo and in vitro /Gutierrez et al. 2007; Ferreira et al. 2009). Targeting of Swi/Snf by activators involves interactions of multiple subunits of Swi/Snf (including Snf2, Snf5, and Swil) with the activator
(Neely et al. 2002; Prochasson et al. 2003). These increased contacts would help stabilize Swi/Snf association with nucleosomes. Interestingly, group 1 and group 3 genes are involved in regulation of translation and metabolic processes that are critical for cell survival and must be constantly transcribed (Supplemental Fig. 1). Although these genes have moderate levels of histone acetylation, the presence of activators would allow for constant recruitment and retention of Swi/Snf, thus minimizing the effects of acetylation of Snf2 (Fig. 9). A subset of these genes (group 1) is down-regulated during stress (Fig. 2A,B). Our analysis shows that this group of genes is involved in translation and ribosome biogenesis (Supplemental Fig. 1). In fact, it is known that activators such as Ifh 2 and Rap1 regulate transcription during normal growth conditions by binding to promoters of ribosomal protein genes. Also, under different stress conditions in yeast, these genes are down-regulated (Gasch et al. 2000; Shivaswamy and Iyer 2008). Stress signals would cause the removal of activator from these genes. In the absence of activator, the acetylated Snf2-containing Swi/Snf would be quickly released, altering nucleosome occupancy at the promoters and allowing genes to turn off (Figs. 2, 5).

Based on our observations, we propose dual modes of regulating Swi/Snf binding and function in genes that can act simultaneously or in parallel (Fig. 9). The inherent

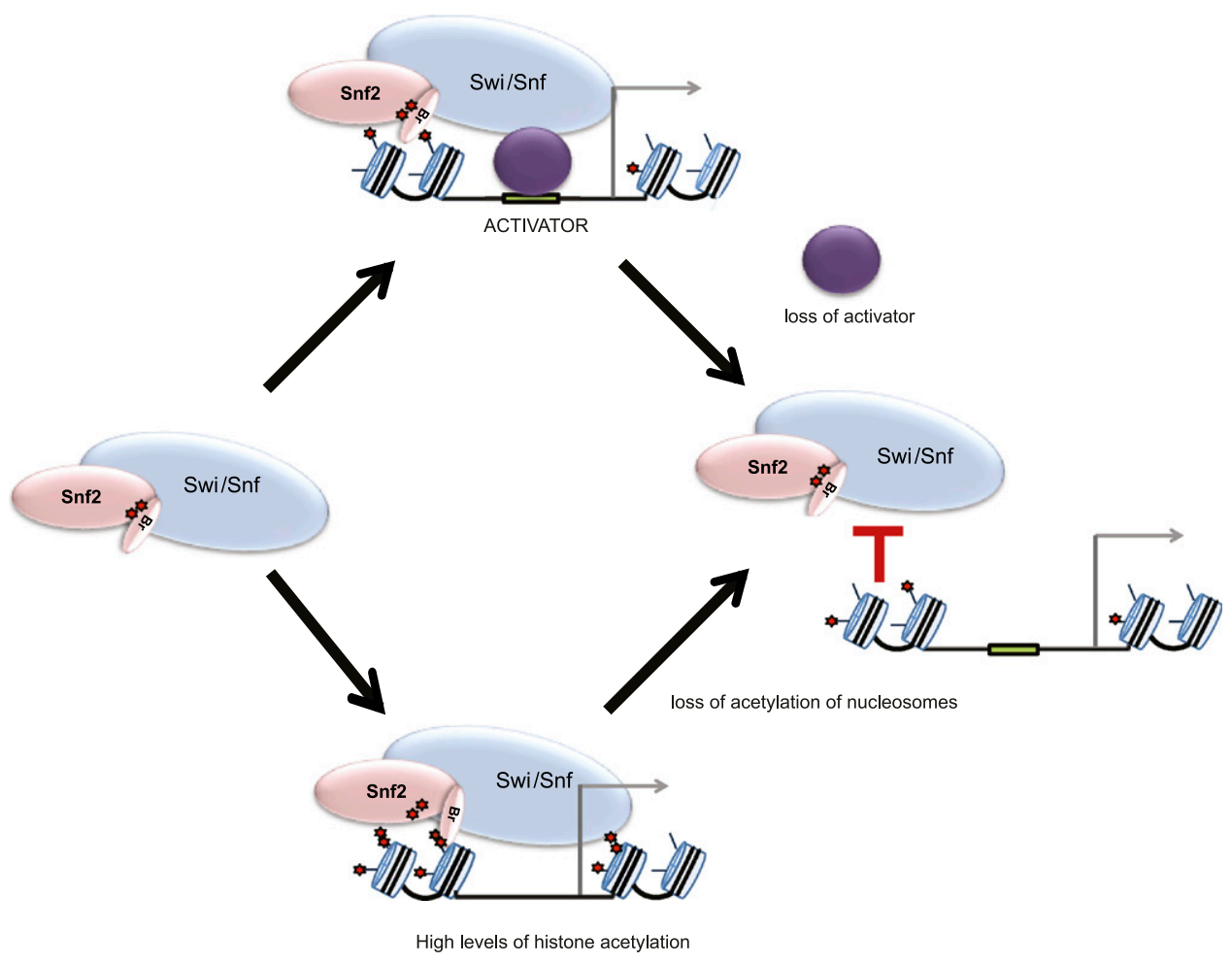

Figure 9. Model for regulation of Swi/Snf recruitment and release from genes. Swi/Snf can be recruited to genes by activators and histone acetylation. High levels of histone acetylation help compete against Snf2 acetylation, allowing for interaction with the bromodomain (group 2 genes). Acting simultaneously or in parallel, activator-dependent recruitment of Swi/Snf can further stabilize Swi/Snf occupancy due to additional contacts with activator by subunits of the Swi/Snf complex that allow for retention of Swi/Snf, keeping genes in an ON state (groups 1, 2, and 3). When genes are turned off, activators are quickly released from promoters of genes (group 1). In the absence of positive regulation by activators and reduction in levels of histone acetylation, decreased competition from acetylated histones allows the bromodomain to bind acetylated residues of Snf2 for limited retention and quick release of Swi/Snf from promoters. 
acetylation of Snf2 and its further increase during stress allow for intramolecular interaction with the bromodomain, preventing aberrant association of Swi/Snf at genomic loci with low levels of histone acetylation. Thus, the bromodomain of Snf2 can act as a sensor for changes in acetylation levels. High levels of histone acetylation in stress-induced genes are needed to outcompete Snf2 acetylation for binding to the bromodomain, which, along with the presence of activator, results in recruitment of Swi/Snf for remodeling of nucleosomes at the promoters. On the other hand, genes important for cell metabolism may be more reliant on the presence of activator, which keeps Swi/Snf constantly occupied at promoters to keep genes in an ON state irrespective of acetylation status of the nucleosomes. Thus, acetylation of Snf2 acts as a molecular switch for the spatio-temporal engagement of the Swi/Snf complex with promoter nucleosomes, aided by activators or histone acetylation. Since the amounts of the Swi/Snf complex are limiting in yeast (100-500 copies per yeast cell), such a mechanism prevents mislocalization of Swi/Snf while providing for an efficient method of recycling Swi/Snf complexes to promoters of genes based on the need for activation.

\section{Materials and methods}

Additional details of the methods are provided in the Supplemental Material.

ChIP-seq

Cells (wild type, Snf2 K1493R K1497R, $\Delta$ bromo, and Snf2 K1493R K1497R $\Delta$ bromo) were grown in YPD to $\mathrm{A} 600=0.8$ and treated or not by growth in YNB $+0.05 \%$ dextrose. ChIP was done from two biological replicates using antibodies against Snf2 (a kind gift from Joseph Reese, Pennsylvania State University) or H3K9Ac (Millipore). Libraries were generated, followed by sequencing on an Illumina platform. ChIP-seq data are accessible through Gene Expression Omnibus (GEO) series accession number GSE61210.

\section{ChIP-seq analysis}

Reads were aligned to yeast genome sacCer2 using bowtie2 (2.1.0) with $-\mathrm{k} 1$. Analysis was done in R (3.0.2) using the packages GenomicRanges (1.14.4), Rsamtools (1.14.2), and rtracklayer (1.22.0). Peaks were called using a custom Perl script run on read count-normalized immunoprecipitation/input ratio tracks for each replicate, requiring a peak of 1.7-fold with a minimum run of 50 bases and a maximum gap of 400 bases. Peaks were intersected with gene transcript start sites $(-700 /+500)$ using BEDTools (2.17.0). Gene groups 1, 2, and 3 were defined by looking for peaks that overlapped the TSSs of genes in wild-type unstressed, 2R unstressed, wild-type stressed, or 2R stressed, respectively. In order to be included, genes had to have peaks overlapping the TSS in both replicates. The heat map was created by hierarchically row-clustering normalized $\log _{2}$ (immunoprecipitation/input) values for regions $-700 /+500$ around the TSSs of genes in groups 1,2, and 3 and combining them into a heat map. Heat map values outside the range of the scale were set to the maximum or minimum value of the scale. The heat map was generated in $\mathrm{R}$ using custom image plotting. One replicate is shown. Line plots were created by extracting normalized immunoprecipitation/input for each gene region, including 500 bases upstream of and downstream from the gene. Signal for these varying size regions was then binned using $\mathrm{R}$ into 80 bins for each gene. Strands were considered, and columns were averaged in order to create an average profile of the given signal for the set of genes in question. Transparent shading around the line indicates the standard error of the mean. $P$-values are based on mean values per gene in the TSS region $(-700 /+500)$ and are from a two-sided Student's $t$-test between samples. Sets of 51 and 73 genes were defined as follows: For the set of 51 genes, a set of genes was found that was in group 1 and in which the sum of normalized $\log _{2}$ immunoprecipitation/input TSS (-700/ +500 ) coverage values was $<0$ in wild type (stressed) and $>0$ in $2 \mathrm{R}$ (stressed). This set was found using input replicate 2 only. For the set of 73 genes, a set of genes was found that was in group 2 and in which the sum of normalized $\log _{2}$ immunoprecipitation/ input TSS $(-700 /+500)$ coverage values was $<0$ in wild type (unstressed) and $>0$ in $2 \mathrm{R}$ (unstressed). This set was found using input replicate 2 only.

\section{Purification of protein complexes}

Swi/Snf complexes were purified using $C^{\prime}$ Flag tag on Snf2 from wild-type, Snf2 K1493R K1497R, and $\Delta g c n 5$ strains. Ada2-TAP complexes and Gal4-VP16 protein were purified as described earlier (Chandy et al. 2006; Gutierrez et al. 2007).

\section{Reconstitution of nucleosomes}

Recombinant histones were purified from E. coli and reconstituted into octamers as described earlier. Mononucleosomes were reconstituted on DNA fragments with a 5' Cy5 label and containing 601 positioning sequence (position of 601 sequence in each template is described in the respective figure legends) (Yun et al. 2012).

\section{Binding and remodeling assays}

The binding of Swi/Snf to DNA or nucleosomes was carried out using fixed amounts of DNA/nucleosomes and titrating increasing amounts of Swi/Snf complexes purified from wild-type, Snf2 K1493R K1497R, and $\Delta g c n 5$ strains followed by native gel electrophoresis and scanning using Typhoon 9400 (GE Healthcare). Remodeling assays were carried out as a function of either time or Swi/Snf complex concentration using fixed concentrations of nucleosomes. Reactions were halted by addition of 750 ng of calf thymus DNA and 500 ng of long oligonucleosomes. Nucleosomes (remodeled or not) were resolved by native PAGE followed by scanning using Typhoon 9400 (GE Healthcare). For assays to study the effect of activator, purified Gal4-VP16 was added as described. Quantitation in each case was done as described in the figure legends using ImageQuant software (GE Healthcare).

\section{ATPase assay}

ATPase assays were carried out using 100 and $200 \mathrm{fmol}$ of Swi/ Snf complex and $100 \mathrm{fmol}$ of DNA or nucleosomes. Reactions were started by the addition of ATP and quenched with EDTA after $30 \mathrm{~min}$. ATP and hydrolyzed Pi were separated on TLC plates and visualized by using a PhosphorImager. Quantitation was done using ImageQuant software (GE Healthcare) to determine the amount of ATP hydrolyzed to ADP and Pi. The picomoles of ATP hydrolyzed was determined by calculating the ratio of released Pi to unhydrolyzed ATP. 
MNase-seq

For MNase-seq analysis, cells (wild type and Snf2 K1493R $\mathrm{K} 1497 \mathrm{R}$ ) were grown in YPD to $\mathrm{A} 600=0.8$ and treated or not by growth in YNB $+0.05 \%$ dextrose. MNase digestion was done on two biological replicates for each sample as described earlier (Rando 2010). Paired-end 50-base reads were aligned to the sacCer2 genome from University of California at Santa Cruz using bowtie2 (2.1.0) with default settings. Genes in groups defined as in ChIP-seq analysis were used to plot TSS plots using 73-base resized and filtered (100- to 200-base fragments only) bam files using ngsplot (2.41.3). Normalized coverage genome browser tracks were generated in $\mathrm{R}$. The midpoint of each fragment sized from 100 to 200 bases was found and then resized to 73 bases, and normalized coverage was generated for each sample by dividing by the total number of alignments for a given sample and multiplying by 1 million. MNase-seq data are accessible through GEO series accession number GSE61210.

\section{Acknowledgments}

We thank Joseph Reese (Pennsylvania State University) for providing Snf2 antibodies, and members of the Workman laboratory for helpful discussions. We acknowledge Malini Natarajan for critical reading of the manuscript. We acknowledge members of the Molecular Biology core at the Stowers Institute for help with library preparation and sequencing. This work was supported by National Institutes of Health grant R01GM047867 to J.L.W. and funding from the Stowers Institute for Medical Research.

\section{References}

Causton HC, Ren B, Koh SS, Harbison CT, Kanin E, Jennings EG, Lee TI, True HL, Lander ES, Young RA. 2001. Remodeling of yeast genome expression in response to environmental changes. Mol Biol Cell 12: 323-337.

Chandy M, Gutierrez JL, Prochasson P, Workman JL. 2006. SWI/ SNF displaces SAGA-acetylated nucleosomes. Eukaryot Cell 5: $1738-1747$.

Charles GM, Chen C, Shih SC, Collins SR, Beltrao P, Zhang X, Sharma T, Tan S, Burlingame AL, Krogan NJ, et al. 2011. Site-specific acetylation mark on an essential chromatinremodeling complex promotes resistance to replication stress. Proc Natl Acad Sci 108: 10620-10625.

Chatterjee N, Sinha D, Lemma-Dechassa M, Tan S, ShogrenKnaak MA, Bartholomew B. 2011. Histone H3 tail acetylation modulates ATP-dependent remodeling through multiple mechanisms. Nucleic Acids Res 39: 8378-8391.

De Winde JH, Crauwels M, Hohmann S, Thevelein JM, Winderickx J. 1996. Differential requirement of the yeast sugar kinases for sugar sensing in establishing the cataboliterepressed state. Eur I Biochem 241: 633-643.

Ferreira R, Eberharter A, Bonaldi T, Chioda M, Imhof A, Becker PB. 2007. Site-specific acetylation of ISWI by GCN5. BMC Mol Biol 8: 73.

Ferreira ME, Prochasson P, Berndt KD, Workman JL, Wright AP. 2009. Activator-binding domains of the SWI/SNF chromatin remodeling complex characterized in vitro are required for its recruitment to promoters in vivo. FEBS / 276: 2557-2565.

Gasch AP, Spellman PT, Kao CM, Carmel-Harel O, Eisen MB, Storz G, Botstein D, Brown PO. 2000. Genomic expression programs in the response of yeast cells to environmental changes. Mol Biol Cell 11: 4241-4257.
Gasch AP, Werner-Washburne M. 2002. The genomics of yeast responses to environmental stress and starvation. Funct Integr Genomics 2: 181-192.

Geng F, Laurent BC. 2004. Roles of SWI/SNF and HATs throughout the dynamic transcription of a yeast glucoserepressible gene. EMBO J 23: 127-137.

Govind CK, Yoon S, Qiu H, Govind S, Hinnebusch AG. 2005. Simultaneous recruitment of coactivators by Gen $4 p$ stimulates multiple steps of transcription in vivo. Mol Cell Biol 25: 5626-5638.

Gutierrez JL, Chandy M, Carrozza MJ, Workman JL. 2007. Activation domains drive nucleosome eviction by SWI/ SNF. EMBO / 26: 730-740.

Hassan AH, Prochasson P, Neely KE, Galasinski SC, Chandy M, Carrozza MJ, Workman JL. 2002. Function and selectivity of bromodomains in anchoring chromatin-modifying complexes to promoter nucleosomes. Cell 111: 369-379.

Hassan AH, Awad S, Prochasson P. 2006. The Swi2/Snf2 bromodomain is required for the displacement of SAGA and the octamer transfer of SAGA-acetylated nucleosomes. I Biol Chem 281: 18126-18134.

Kim JH, Saraf A, Florens L, Washburn M, Workman JL. 2010. Gcn5 regulates the dissociation of SWI/SNF from chromatin by acetylation of Swi2/Snf2. Genes Dev 24: 2766-2771.

Lee KK, Workman JL. 2007. Histone acetyltransferase complexes: one size doesn't fit all. Nat Rev Mol Cell Biol 8: 284-295.

Lerin C, Rodgers JT, Kalume DE, Kim SH, Pandey A, Puigserver P. 2006. GCN5 acetyltransferase complex controls glucose metabolism through transcriptional repression of PGC- $1 \alpha$. Cell Metab 3: 429-438.

Neely KE, Hassan AH, Brown CE, Howe L, Workman JL. 2002. Transcription activator interactions with multiple SWI/SNF subunits. Mol Cell Biol 22: 1615-1625.

Peterson CL, Workman JL. 2000. Promoter targeting and chromatin remodeling by the SWI/SNF complex. Curr Opin Genet Dev 10: 187-192.

Prochasson P, Neely KE, Hassan AH, Li B, Workman JL. 2003. Targeting activity is required for SWI/SNF function in vivo and is accomplished through two partially redundant activator-interaction domains. Mol Cell 12: 983-990.

Rando OJ. 2010. Genome-wide mapping of nucleosomes in yeast. Methods Enzymol 470: 105-118.

Roberts SM, Winston F. 1997. Essential functional interactions of SAGA, a Saccharomyces cerevisiae complex of Spt, Ada, and Gcn5 proteins, with the Snf/Swi and Srb/mediator complexes. Genetics 147: 451-465.

Shivaswamy S, Iyer VR. 2008. Stress-dependent dynamics of global chromatin remodeling in yeast: dual role for SWI/SNF in the heat shock stress response. Mol Cell Biol 28: 22212234.

Singh M, D'Silva L, Holak TA. 2006. DNA-binding properties of the recombinant high-mobility-group-like AT-hook-containing region from human BRG1 protein. Biol Chem 387: 14691478.

Sudarsanam P, Winston F. 2000. The Swi/Snf family nucleosome-remodeling complexes and transcriptional control. Trends Genet 16: 345-351.

Sudarsanam P, Iyer VR, Brown PO, Winston F. 2000. Wholegenome expression analysis of snf/swi mutants of Saccharomyces cerevisiae. Proc Natl Acad Sci 97: 3364-3369.

Tachibana C, Yoo JY, Tagne JB, Kacherovsky N, Lee TI, Young ET. 2005. Combined global localization analysis and transcriptome data identify genes that are directly coregulated by Adr1 and Cat8. Mol Cell Biol 25: 2138-2146. 
Dutta et al.

VanDemark AP, Kasten MM, Ferris E, Heroux A, Hill CP, Cairns BR. 2007. Autoregulation of the rsc 4 tandem bromodomain by gen5 acetylation. Mol Cell 27: 817-828.

Yen K, Vinayachandran V, Batta K, Koerber RT, Pugh BF. 2012. Genome-wide nucleosome specificity and directionality of chromatin remodelers. Cell 149: 1461-1473.

Yun M, Ruan C, Huh JW, Li B. 2012. Reconstitution of modified chromatin templates for in vitro functional assays. Methods Mol Biol 833: 237-253.

Zhang H, Kruk JA, Reese JC. 2008. Dissection of coactivator requirement at RNR3 reveals unexpected contributions from TFIID and SAGA. J Biol Chem 283: 27360-27368. 


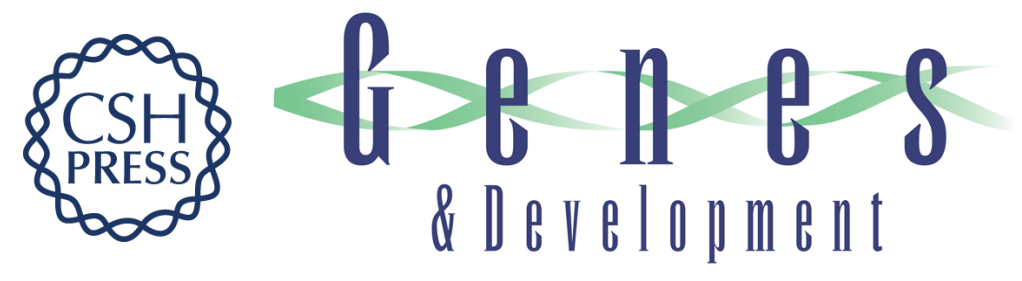

\section{Swi/Snf dynamics on stress-responsive genes is governed by competitive bromodomain interactions}

Arnob Dutta, Madelaine Gogol, Jeong-Hoon Kim, et al.

Genes Dev. 2014, 28:

Access the most recent version at doi:10.1101/gad.243584.114

\section{Supplemental Material \\ http://genesdev.cshlp.org/content/suppl/2014/10/15/28.20.2314.DC1}

References

This article cites 31 articles, 15 of which can be accessed free at: http://genesdev.cshlp.org/content/28/20/2314.full.html\#ref-list-1

Creative

Commons

License

Email Alerting
Service

This article is distributed exclusively by Cold Spring Harbor Laboratory Press for the first six months after the full-issue publication date (see

http://genesdev.cshlp.org/site/misc/terms.xhtml). After six months, it is available under a Creative Commons License (Attribution-NonCommercial 4.0 International), as described at http://creativecommons.org/licenses/by-nc/4.0/.

Receive free email alerts when new articles cite this article - sign up in the box at the top right corner of the article or click here.

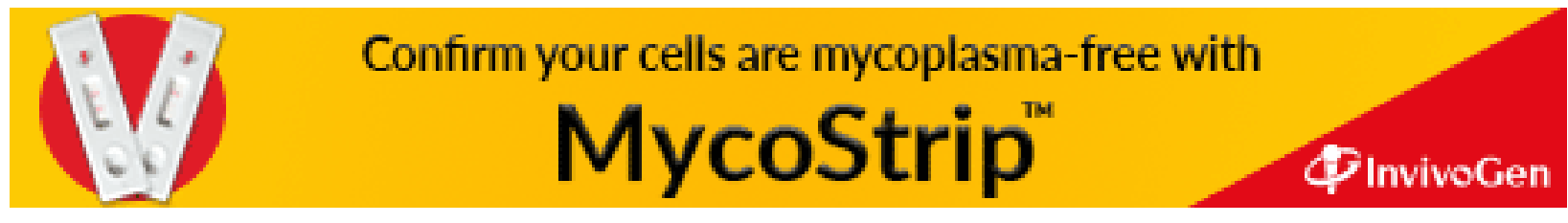

